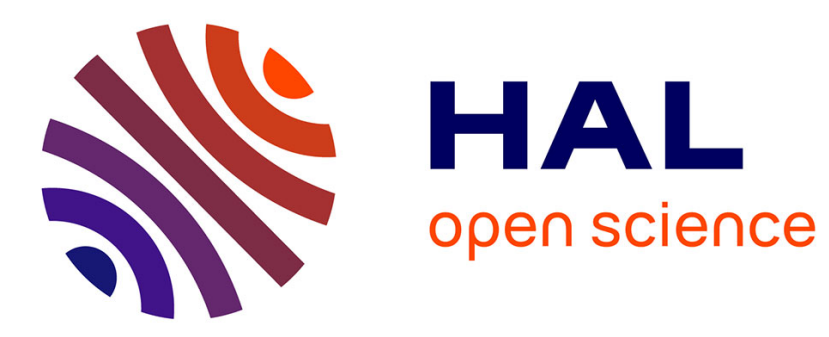

\title{
Convolution in (max,min)-algebra and its role in mathematical morphology
}

Jesus Angulo

\section{To cite this version:}

Jesus Angulo. Convolution in (max,min)-algebra and its role in mathematical morphology. Advances in Imaging and Electron Physics, 2017, 203, pp.1-66. 10.1016/bs.aiep.2017.07.003 . hal-01108121

\section{HAL Id: hal-01108121}

https://hal-mines-paristech.archives-ouvertes.fr/hal-01108121

Submitted on 22 Jan 2015

HAL is a multi-disciplinary open access archive for the deposit and dissemination of scientific research documents, whether they are published or not. The documents may come from teaching and research institutions in France or abroad, or from public or private research centers.
L'archive ouverte pluridisciplinaire HAL, est destinée au dépôt et à la diffusion de documents scientifiques de niveau recherche, publiés ou non, émanant des établissements d'enseignement et de recherche français ou étrangers, des laboratoires publics ou privés. 


\title{
Convolution in (max, min)-algebra and its role in mathematical morphology
}

\author{
Jesús Angulo \\ MINES ParisTech, PSL-Research University, \\ CMM-Centre de Morphologie Mathématique; France \\ jesus.angulo@mines-paristech.fr
}

September 2014

\begin{abstract}
The purpose of this theoretical paper is to study the convolution of two functions in the (max, min)-algebra. More precisely, a formal definition of morphological operators in (max, min)-algebra is introduced and their relevant properties from an algebraic viewpoint are stated and proved. Some previous works in mathematical morphology have already encountered this type of operators but a systematic study of them has not yet been undertaken in the morphological literature. It is shown in particular that their fundamental property is the equivalence with level set processing using Minkowski addition and subtraction. Some powerful results from nonlinear analysis can be straightforward related to the present (max, $\min$ )-operators. On the one hand, the theory of viscosity solutions of the Hamilton-Jacobi equation with Hamiltonians containing $u$ and $D u$ is summarized, in particular, the corresponding Hopf-Lax-Oleinik formulas are given. On the other hand, results on quasi-concavity preservation, Lipschitz approximation and conjugate/tranform related to $(\max , \min )$-convolutions are discussed. Links between (max, min)-convolutions and some previous approaches of unconventional morphology, in particular fuzzy morphology and viscous morphology, are fully reviewed. In addition, the interest of $(\max , \min )$-convolutions in Boolean random function characterization is considered. Links of (max, min)-morphology framework to geodesic dilation and erosion are also provided. We discuss two important conclusions. First, it is proven in the paper that (max, min)-openings are compatible with Matheron's axiomatic of Euclidean granulometries for functions with quasiconcave structuring functions. Second, it is also shown that the adjoint supmin convolution is the operator underlying the extension of Matheron's characterization of Boolean random closed sets to the case of Boolean random upper semicontinuous function. For all these reasons, we state that ( $\max , \min$ )-convolution provides the natural framework to generalize some key notions from Matheron's theory from sets to functions.
\end{abstract}


Keywords: mathematical morphology; (max, min)-algebra ; level set addition ; adjunction ; duality ; Hamilton-Jacobi PDE ; quasiconvexity ; Lipschitz regularization ; fuzzy morphology ; viscous morphology; random upper semicontinuous functions; Choquet capacity for functions.

\section{Contents}

1 Introduction $\quad 3$

2 Basic notions and notations 5

3 (max, $\min$ )-convolutions: definition and properties 9

3.1 Definition . . . . . . . . . . . . . . . . . . . 9 9

3.2 Duality by complement vs. duality by adjunction . . . . . . . . . . . 10

3.3 Commutation with level set processing . . . . . . . . . . . . . . . 13

3.4 Further properties . . . . . . . . . . . . . . . 16

3.5 Openings, closings using (max, min)-convolutions and granulometries . . . . . 19

4 Hopf-Lax-Oleinik formulas for Hamilton-Jacobi equation $u_{t} \pm H(u, D u)=0 \mathbf{2 4}$

4.1 Morphological PDE for classical dilation and erosion . . . . . . . . . . 24

4.2 Viscosity solution of Hamilton-Jacobi equation with Hamiltonians containing

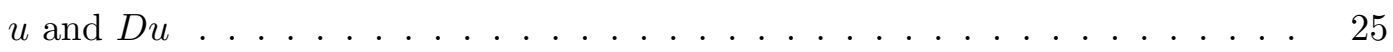

5 Nonlinear analysis using operators $(\triangle, \nabla) \quad 29$

5.1 Quasi-concavity . . . . . . . . . . . . . . . . . . . 29

5.2 Lipschitz approximation . . . . . . . . . . . . . . . . . . 31

5.3 A transform in $(\max , \min )$-convolution $\ldots \ldots \ldots \ldots . \ldots . \ldots . \ldots 34$

6 Ubiquity of (max, min)-convolutions in mathematical morphology 39

6.1 Distance function . . . . . . . . . . . . . . . . . . . . 39

6.2 Flat morphological operators using indicator functions . . . . . . . . . . 39

6.3 Links with fuzzy morphology . . . . . . . . . . . . . . . . . . . 41

6.4 Links with viscous morphology . . . . . . . . . . . . . . . . . . . . . . . . 42

6.5 Links with Boolean random function characterization . . . . . . . . . . . . . 44

6.6 Links with geodesic dilation and erosion . . . . . . . . . . . . . 50

7 Conclusion and Perspectives $\quad 52$ 


\section{Introduction}

Pioneered for Boolean random sets [46], extended later to grey-level images [58] and more generally formulated in the framework of complete lattices [59, 29], mathematical morphology is a nonlinear image processing methodology useful for efficiently solving many image analysis tasks [62].

Let $E$ be the Euclidean $\mathbb{R}^{n}$ or discrete space $\mathbb{Z}^{n}$ (support space) and let $\mathcal{T}$ be a set of greylevels (space of values). For theoretical reasons it is assumed that $\mathcal{T}=\overline{\mathbb{R}}=\mathbb{R} \cup\{-\infty,+\infty\}$, but one often has $\mathcal{T}=[0, M]$. A grey-level image is represented by a function $f: E \rightarrow \mathcal{T}$, also noted as $f \in \mathcal{F}(E, \overline{\mathbb{R}})$, such that $f$ maps each pixel $x \in E$ into a grey-level value in $\mathcal{T}$. Given a grey-level image, the two basic morphological mappings $\mathcal{F}(E, \mathcal{T}) \rightarrow \mathcal{F}(E, \mathcal{T})$ are the dilation and the erosion given respectively by

$$
\left\{\begin{array}{c}
(f \oplus b)(x)=\sup _{y \in E}\{f(y)+b(x-y)\}, \\
(f \ominus b)(x)=\inf _{y \in E}\{f(y)-b(y-x)\},
\end{array}\right.
$$

where $b \in \mathcal{F}(E, \mathcal{T})$ is the structuring function which determines the effect of the operator. The other morphological operators, such as the opening and the closing, are obtained by composition of dilation/erosion [58, 29]. The Euclidean framework has been recently generalized to images supported on Riemannian manifolds [6].

If we compare the mathematical structure of the operators (1) to the classical convolution of a function $f$ by a kernel $k$, let say the convolution in the $(+, \times)$-algebra; i.e.,

$$
(f * k)(x)=\int_{E} f(y) k(x-y) d y,
$$

then, we can establish in parallelism of the involved mathematical operations. Thus, operators (1) are interpreted in nonlinear mathematics as the convolution in ( $\max ,+)$-algebra (and in its dual algebra) [27]. More precisely, the study of the operator equivalent to the erosion (1) can be traced back to the notion of inf-convolution (or infimal convolution) introduced by Moreau in the 70's of last century [49], as the fundamental tool in convex analysis. Convolution in $(\max ,+)$-algebra has been also widely studied in the framework of idempotent analysis developed by Maslov and co-workers [34]. This inherent connection of functional operators (1) with the supremal and infimal convolution of nonlinear mathematics and convex analysis has been extremely fruitful to the state-of-the-art on mathematical morphology. Morphological PDEs $[1,7,17,42]$, the slope transform [22, 41], or more recently to the notion of stochastic morphology based on Maslov random walks [5], are particularizations of results from nonlinear mathematics.

Nevertheless, the functional operators (1) do not extend all the fundamental properties of the dilation and erosion for sets, as formulated in Matheron's theory. Perhaps the most disturbing for us are, on the one hand, the lack of commutation with level set processing for nonflat structuring functions; on the other hand, the limitation of Matheron's axiomatic of 
granulometry to constant (i.e., flat) functions on a convex domain [35]. In addition, there are some unconventional morphological frameworks, such as the fuzzy morphology $[50,20,44,14]$ or the viscous morphology $[63,64,45]$ which do not fit in the classical (max, +)-algebra framework.

Actually, the $(\max ,+)$ is not the unique possible alternative to see morphological operators as convolutions. The idea in this paper is to consider the operation of convolution of two functions in the ( $\max , \min )$-algebra. This is in fact our main motivation: to formally introduce the notion of (max, min)-mathematical morphology. As we show in the paper, this framework is not totally new in morphology since some fuzzy morphological operators are exactly the same convolutions that we introduce. But some of the key properties are ignored by in the fuzzy context, and the most important, they are not limited to fuzzy sets. By the way, even if much less considered than the supremal and infimal convolutions, convolutions in ( $\max , \min )$-algebra have been the object of various studies in different branches of nonlinear applied mathematics, from quasi-convex analysis $[67,57,68,26,39,53]$ to viscosity solutions of Hamilton-Jacobi equations [11, 12, 2, 65]. Interested reader is also referred to the book [27] for a systematic comparative study of matrix algebra and calculus in the three algebras $(+, \times),(\max ,+)$ and $(\max , \min )$, and to the book [18] for a nonlinear partial differential equations viewpoint. Results from this literature are extremely useful for us. Indeed, convolution in (max, min)-algebra is known in convex analysis literature as "sublevel convolution" or " "level sets sum convolution". The reason for this name is obvious: the fundamental property of convolution in (max, min)-algebra is the commutation with level set processing. In fact, this principle is the inspiration and second motivation of this work: to show how (max, min)-mathematical morphology can covers some of the unconventional operators which are formalized by level set processing.

The present work is exclusively a theoretical study and thus the practical interest of the operators is not illustrated here.

Paper organization. The rest of the paper is organized as follows.

- Section 2 gives an overview to basic notions on classical mathematical morphology and fixes the notation.

- A formal definition of morphological operators in (max, min)-algebra is introduced in Section 3. Relevant properties from an algebraic viewpoint are stated and proved.

- Sections 4 and 5 provide an overview of nonlinear analysis in (max, min) mathematics and can be straightforward related to our ( $\max , \min$ )-convolutions. On the one hand, the theory of viscosity solutions of the Hamilton-Jacobi equation with Hamiltonians containing $u$ and $D u$ is summarized, in particular, the corresponding Hopf-Lax-Oleinik formulas are given in Section 4. Section 5 discusses the results on quasi-concavity preservation, Lipschitz approximation and conjugate/tranform related to (max, min)convolutions. 
- Section 6 reviews the links between (max, min)-convolutions and some previous approaches of unconventional morphology, in particular fuzzy morphology and viscous morphology. In addition, the interest of ( $\max , \min )$-convolutions in Boolean random function characterization is considered. Links of (max, min) framework to geodesic dilation and erosion are also provided.

- Section 7 of conclusion and perspectives closes the paper.

\section{Basic notions and notations}

For more details on this background material, see classical references on set morphology [46], on flat morphology for functions [58](Chapter XII) and [40] or on complete lattice formulation of morphological operators [59] [29].

Minkowski addition and subtraction. Given a set $X \subseteq E$, the complement of $X$ is $X^{c}=E \backslash X$, and the transpose of $X$ (or symmetrical set with respect to the origin $O$ ) is $\check{X}=$ $\{-x: x \in X\}$. For every $p \in E$, the translate of $X$ by $p$ is $X_{p}=\{x+p: x \in X\}$. For any pair of sets $X$ and $Y$, their Minkowski addition $\oplus$ and Minkowski subtraction $\ominus$ are defined as follows:

$$
\begin{aligned}
& X \oplus Y=\bigcup_{y \in Y} X_{y}=\{x+y: x \in X, y \in Y\}=\left\{p \in E: X \cap \check{Y}_{p} \neq \emptyset\right\}, \\
& X \ominus Y=\bigcap_{y \in Y} X_{-y}=\left\{p \in E: Y_{p} \subset X\right\}=\left\{x: \forall p \in \check{Y}, x \in X_{p}\right\} .
\end{aligned}
$$

We remind that the (binary) dilation and erosion of set $X$ by structuring element $B, B \subseteq E$, are just defined respectively as

$$
\delta_{B}(X)=X \oplus B \text { and } \quad \varepsilon_{B}(X)=X \ominus B .
$$

It should be noted that dilation and erosion are dual by complementation:

$$
X \oplus B=\left(X^{c} \ominus \check{B}\right)^{c} \quad \text { and } \quad X \ominus B=\left(X^{c} \oplus \check{B}\right)^{c} .
$$

It is sometimes misunderstandood that duality by complement is the right way to construct erosion from dilation and viceversa. There is however another duality in mathematical morphology which is just indicated for such purpose: the duality by adjunction, which in the case of binary operators involves

$$
X \oplus B \subseteq Y \quad \Leftrightarrow \quad X \subseteq Y \ominus B
$$

Composition of dilation and erosion leads to two extremely powerful morphological operators. Namely, the opening and the closing of set $X$ by $B$, which are defined respectively 


$$
\begin{aligned}
X \circ B & =(X \ominus B) \oplus B, \\
X \bullet B & =(X \oplus B) \ominus B .
\end{aligned}
$$

Opening is anti-extensive and closing is extensive. But the most important property of the opening and closing operators is their idempotence; that is, their stability to iteration.

Erosion, dilation and adjunction in complete lattices. Morphological operators are formulated in a more abstract framework based on complete lattice theory. We briefly recall this framework.

Let us consider a nonempty set $\mathcal{L}$ endowed with a partial ordering $\leq$. We say that $(\mathcal{L}, \leq)$ is a complete lattice if every subset $X \subset \mathcal{L}$ has an infimum $\bigwedge X$ and a supremum $\bigvee X$ in $\mathcal{L}$. The least and the greatest elements of a complete lattice are denoted respectively by $\perp$ and $\top$.

The operators $\varepsilon: \mathcal{L} \rightarrow \mathcal{L}$ and $\delta: \mathcal{L} \rightarrow \mathcal{L}$ are called an erosion and a dilation if they commute respectively with the infimum and the supremum; i.e., $\varepsilon\left(\bigwedge_{i \in I} X_{i}\right)=\bigwedge_{i \in I} \varepsilon\left(X_{i}\right)$ and $\delta\left(\bigvee_{i \in} X_{i}\right)=\bigvee_{i \in} \delta\left(X_{i}\right)$, for every collection $\left\{X_{i}: i \in\right\} \subset \mathcal{L}$. Erosion and dilation are increasing operators, i.e., $\forall X, Y \in \mathcal{L}$, if $X \leq Y$ then $\varepsilon(X) \leq \varepsilon(Y)$ and $\delta(X) \leq \delta(Y)$. Erosion and dilation are related by the notion of adjunction:

$$
\delta(X) \leq Y \Leftrightarrow X \leq \varepsilon(Y) ; \forall X, Y \in \mathcal{L}
$$

Adjunction law (6) is of fundamental importance in mathematical morphology since it allows to define a unique dilation $\delta$ associated to a given erosion $\varepsilon$, i.e.,

$$
\delta(X)=\bigwedge\{Y \in \mathcal{L}: X \leq \varepsilon(Y)\} ; X \in \mathcal{L} .
$$

Similarly one can define a unique erosion from a given dilation:

$$
\varepsilon(Y)=\bigvee\{X \in \mathcal{L}: \delta(X) \leq Y\} ; Y \in \mathcal{L} .
$$

Given an adjunction $(\varepsilon, \delta)$, their composition product operators, $\gamma(X)=\delta(\varepsilon(X))$ and $\varphi(Y)=$ $\varepsilon(\delta(Y))$ are respectively an opening and a closing, which are the basic morphological filters having the following properties: idempotency $\gamma \gamma(X)=\gamma(X)$, anti-extensivity $\gamma(X) \leq X$ and extensivity $X \leq \varphi(X)$, and increaseness. Another relevant result is the fact that, given an erosion $\varepsilon$, the opening and closing by adjunction are exclusively defined in terms of this erosion as

$$
\begin{aligned}
\gamma(X) & =\bigwedge\{Y \in \mathcal{L}: \varepsilon(X) \leq \varepsilon(Y)\}, \quad \forall X \in \mathcal{L}, \\
\varphi(X) & =\bigwedge\{\varepsilon(Y): Y \in \mathcal{L}, X \leq \varepsilon(Y)\}, \quad \forall X \in \mathcal{L} .
\end{aligned}
$$


Dilation and erosion for functions and links with level set processing. As mentioned above, the dilation and erosion of a function $f \in \mathcal{F}(E, \overline{\mathbb{R}})$, by a structuring function $b \in \mathcal{F}(E, \overline{\mathbb{R}})$ are defined as

$$
\delta_{b}(f)(x)=(f \oplus b)(x) \text { and } \varepsilon_{b}(f)(x)=(f \ominus b)(x) .
$$

where $(f \oplus b)$ and $(f \ominus b)$ were given in (1).

Dilation and erosion are dual by adjunction, i.e.,

$$
f \oplus b \leq g \quad \Leftrightarrow \quad f \leq g \ominus b
$$

as well as dual by complement, i.e.,

$$
\left(f^{c} \oplus b\right)^{c}=f \ominus \check{b} \quad \text { and } \quad\left(f^{c} \ominus b\right)^{c}=f \oplus \check{b},
$$

where the complement function $\phi^{c}$ of function $\phi$ is defined as the negation for real valued functions and the symmetric with respect to $M$ for function valued in a nonnegative interval $[0, M]$, i.e., $\phi^{c}(x)=-\phi(x)$ if $\phi \in \mathcal{F}(E, \overline{\mathbb{R}})$ or $\phi^{c}(x)=M-\phi(x)$ if $\phi \in \mathcal{F}(E,[0, M])$. The transposed function $\check{\phi}$ is given by $\check{\phi}(x)=\phi(-x)$.

The structuring function is usually a parametric multiscale family $b_{\lambda}(x)$, where $\lambda>0$ is the scale parameter such that $b_{\lambda}(x)=\lambda b(x / \lambda)$ and which satisfies the semi-group property $\left(b_{\lambda} \oplus b_{\mu}\right)(x)=b_{\lambda+\mu}(x)$.

The most commonly studied framework for dilation/erosion of functions, which additionally presents better properties of invariance, is based on flat structuring functions, therefore viewed as structuring elements. More precisely, given the structuring element $B \subseteq E$, its associated structuring function is

$$
b(x)=\left\{\begin{array}{cc}
0 & \text { if } x \in B \\
-\infty & \text { if } x \in B^{c}
\end{array}\right.
$$

Hence, the flat dilation $(f \oplus B)$ and flat erosion $(f \ominus B)$ can be computed respectively by the moving local maxima and minima filters; i.e., $(f \oplus B)(x)=\sup _{y \in \check{B}} f(x+y)$ and $(f \ominus B)(x)=$ $\inf _{y \in B} f(x+y)$.

Given an upper semicontinuous (USC) function $f \in \mathcal{F}(E, \overline{\mathbb{R}})$, it can be defined by means of its upper level sets $X_{h}^{+}(f)$ as follows

$$
f(x)=\sup \left\{h \in \overline{\mathbb{R}}: x \in X_{h}^{+}(f)\right\},
$$

or if $f$ is lower semicontinuous (LSC), using its lower level sets $X_{h}^{-}(f)$, as

$$
f(x)=\inf \left\{h \in \overline{\mathbb{R}}: x \in X_{h}^{-}(f)\right\} .
$$


We note that a USC function can be also represented as

$$
\begin{aligned}
f(x) & =\inf \left\{h \in \overline{\mathbb{R}}: x \notin X_{h}^{+}(f)\right\} \\
& =\inf \left\{h \in \overline{\mathbb{R}}: x \in Y_{h}^{-}(f)\right\},
\end{aligned}
$$

similarly a LSC function can be represented as

$$
f(x)=\sup \left\{h \in \overline{\mathbb{R}}: x \in Y_{h}^{+}(f)\right\},
$$

where

$$
\begin{aligned}
& X_{h}^{+}(f)=\{x \in E: f(x) \geq h\}, \quad \text { and } \quad Y_{h}^{+}(f)=\{x \in E: f(x)>h\} ; \\
& X_{h}^{-}(f)=\{x \in E: f(x) \leq h\}, \quad \text { and } \quad Y_{h}^{-}(f)=\{x \in E: f(x)<h\} .
\end{aligned}
$$

Considering $E \subset \overline{\mathbb{R}}^{n}$, the upper level sets $X_{t}^{+}$of any $F$ are closed sets in $\overline{\mathbb{R}}^{n}$, decreasing, i.e., $h<k \Rightarrow X_{k}^{+} \subseteq X_{h}^{+}$and obey the monotonic continuity $X_{k}^{+}=\cap_{h<k} X_{h}^{+}$. Lower level sets are closed sets, increasing $h<k \Rightarrow X_{h}^{-} \subseteq X_{k}^{-}$and $X_{k}^{-}=\cup_{h<k} X_{h}^{-}$. Obviously, a continuous function $f$ can be decomposed/reconstructed using either its (strict) upper level sets or its (strict) lower level sets. We note that, using duality by complement, one has

$$
\left(X_{h}^{+}(f)\right)^{c}=Y_{h}^{-}(f)
$$

and thus the strict lower sets $Y_{h}^{-}(f)$ are open sets. We note also that a sampled function is trivially both USC and LSC because all its level sets are both closed and open sets.

As mentioned before, flat operators commute with level set processing. Being more precise, first notice that for any collection of continuous functions $\phi_{i} \in \mathcal{F}(E, \overline{\mathbb{R}}), i \in I$, we have:

$$
\begin{aligned}
& X_{h}^{+}\left(\bigvee_{i} \phi_{i}\right)=\left\{x \in E: \phi_{1}(x) \geq h \text { or } \phi_{2}(x) \geq h \text { or } \cdots\right\}=\bigcup_{i} X_{h}^{+}\left(\phi_{i}\right) \\
& X_{h}^{+}\left(\bigwedge_{i} \phi_{i}\right)=\left\{x \in E: \phi_{1}(x) \geq h \text { and } \phi_{2}(x) \geq h \text { and } \cdots\right\}=\bigcap_{i} X_{h}^{+}\left(\phi_{i}\right)
\end{aligned}
$$

and by (9), their dual expressions by complement:

$$
\begin{aligned}
& Y_{h}^{-}\left(\bigvee_{i} \phi_{i}\right)=\left\{x \in E: \phi_{1}(x)<h \text { and } \phi_{2}(x)<h \text { and } \cdots\right\}=\bigcap_{i} Y_{h}^{-}\left(\phi_{i}\right), \\
& Y_{h}^{-}\left(\bigwedge_{i} \phi_{i}\right)=\left\{x \in E: \phi_{1}(x)<h \text { or } \phi_{2}(x)<h \text { or } \cdots\right\}=\bigcup_{i} Y_{h}^{-}\left(\phi_{i}\right) .
\end{aligned}
$$

The following formulas [39], relating lower level sets and strict lower level sets, are also useful in the sequel:

$$
\begin{aligned}
X_{h}^{-}(f) & =\bigcap_{k>h} Y_{k}^{-}(f), \\
Y_{h}^{-}(f) & =\bigcup_{j=1}^{\infty} X_{h-1 / j}^{-}(f) .
\end{aligned}
$$


where the family $\left\{X_{h-(1 / j)}^{-}(f)\right\}, j \geq 1$, is increasing.

Then, taking for $\phi_{i}$ in (10) and (11) all the translates of $f$ as $x$ runs over a set $B$ or its transpose, together with definitions of $\oplus$ and $\ominus$, respectively (2) and (3), we can write:

$$
\begin{aligned}
& X_{h}^{+}(f \oplus B)=\left\{x \in E: \exists y \in B_{x}, f(y) \geq h\right\}=X_{h}^{+}(f) \oplus B, \\
& X_{h}^{+}(f \ominus B)=\left\{x \in E: \forall y \in \check{B}_{x}, f(y) \geq h\right\}=X_{h}^{+}(f) \ominus B .
\end{aligned}
$$

This property of commutativity of upper level sets involves that the flat dilation and erosion of a continuous function $f \in \mathcal{F}(E, \overline{\mathbb{R}})$ by structuring element $B \subset E$ are obtained as:

$$
\begin{aligned}
& \delta_{B}(f)(x)=\sup \left\{h \in \overline{\mathbb{R}}: x \in\left(X_{h}^{+}(f) \oplus B\right)\right\}, \\
& \varepsilon_{B}(f)(x)=\sup \left\{h \in \overline{\mathbb{R}}: x \in\left(X_{h}^{+}(f) \ominus B\right)\right\} .
\end{aligned}
$$

For the opening and closing of a function $f$ by a flat structuring element, denoted $\gamma_{B}(f)$ and $\varphi_{B}(f)$, and defined by

$$
\begin{aligned}
\gamma_{B}(f) & =f \circ B=(f \ominus B) \oplus B), \\
\varphi_{B}(f) & =f \bullet B=(f \oplus B) \ominus B),
\end{aligned}
$$

we also have a natural formulation using upper level sets:

$$
\begin{aligned}
& X_{h}^{+}(f \circ B)=X_{h}^{+}(f) \circ B, \\
& X_{h}^{+}(f \bullet B)=X_{h}^{+}(f) \bullet B .
\end{aligned}
$$

In the case of unflat dilation and erosion of function $f$ by structuring function $b$, their formulation using sets is not very useful since it needs all the upper level sets:

$$
\begin{aligned}
& X_{k}^{+}(f \oplus b)=\bigcup_{h}\left[X_{h}^{+}(f) \oplus X_{k-h}^{+}(b)\right], \\
& X_{k}^{+}(f \ominus b)=\bigcap_{h}\left[X_{h}^{+}(f) \ominus X_{h-k}^{+}(b)\right] .
\end{aligned}
$$

\section{3 (max, min)-convolutions: definition and properties}

In this Section we define the alternative convolutions associated to a pair (function $f$, structuring function $b$ ) in the ( $\max , \min )$ mathematical framework. We also study their properties.

\subsection{Definition}

Definition 1 Given a structuring function $b \in \mathcal{F}\left(\mathbb{R}^{n}, \overline{\mathbb{R}}\right)$, for any function $f \in \mathcal{F}\left(\mathbb{R}^{n}, \overline{\mathbb{R}}\right)$ we define the supmin convolution $f \nabla b$ and the infmax convolution $f \triangle b$ of $f$ by $b$ as

$$
(f \nabla b)(x)=\sup _{y \in \mathbb{R}^{n}}\{f(y) \wedge b(x-y)\}
$$


and

$$
(f \triangle b)(x)=\inf _{y \in \mathbb{R}^{n}}\left\{f(y) \vee b^{c}(y-x)\right\} .
$$

We also define the adjoint infmax $f \triangle^{*} b$ and the adjoint supmin $f \nabla^{*} b$ convolutions as

$$
\left(f \triangle^{*} b\right)(x)=\inf _{y \in \mathbb{R}^{n}}\left\{f(y) \wedge^{*} b(y-x)\right\},
$$

and

$$
\left(f \nabla^{*} b\right)(x)=\sup _{y \in \mathbb{R}^{n}}\left\{f(y) \vee^{*} b^{c}(x-y)\right\},
$$

where $\wedge^{*}$ is the adjoint operator to the minimum $\wedge$ and is given by

$$
f(y) \wedge^{*} b(y-x)=\left\{\begin{array}{cl}
f(y) & \text { if } b(y-x)>f(y) \\
\top & \text { if } b(y-x) \leq f(y)
\end{array}\right.
$$

and $\vee^{*}$ the adjoint to $\vee$ :

$$
f(y) \vee^{*} b^{c}(x-y)=\left\{\begin{array}{cl}
f(y) & \text { if } b^{c}(x-y)<f(y) \\
\perp & \text { if } b^{c}(x-y) \geq f(y)
\end{array}\right.
$$

and where, if we define $\max g=\sup _{x \in \mathbb{R}^{n}} g(x)$ and $\min g=\inf _{x \in \mathbb{R}^{n}} g(x)$, the top and bottom elements for pair of functions $f$ and $b$ correspond to

$$
\top=(\max f) \vee(\max b) \quad \text { and } \quad \perp=(\min f) \wedge\left(\min b^{c}\right) .
$$

Definitions remain valid if we replace $\mathbb{R}^{n}$ by a subset $E$ or any subset of discrete space $\mathbb{Z}^{n}$. Similarly, the extended real line $\overline{\mathbb{R}}$ can be replaced by a bounded, eventually discrete, set of intensities $[0, M]$.

Figure 1 illustrates the four ( $\max , \min )$-convolutions for a given example of one dimensional functions defined in a bounded interval, i.e., $f, b \in \mathcal{F}(\mathbb{R},[0, M])$.

The fact that four convolution operators are naturally defined in ( $\max , \min )$-algebra is related to the previous discussion on dualities by complement and by adjunction and is easily justified by the following property.

\subsection{Duality by complement vs. duality by adjunction}

It is obvious that the four operators are translation invariant. Furthermore, from a morphological viewpoint, their most salient properties are summarized in this proposition.

Proposition 2 The supmin convolution $\nabla$ and infmax convolution $\triangle$ are dual with respect to the complement. Similarly, the adjoint infmax convolution $\triangle^{*}$ and the adjoint supmin $\nabla^{*}$ convolution are dual with respect to the complement, i.e., for $f, b \in \mathcal{F}\left(\mathbb{R}^{n}, \overline{\mathbb{R}}\right)$ one has

$$
\begin{array}{ccc}
f \triangle b=\left(f^{c} \nabla \check{b}\right)^{c} & \text { and } & f \nabla b=\left(f^{c} \triangle \check{b}\right)^{c} \\
f \triangle^{*} b=\left(f^{c} \nabla^{*} \check{b}\right)^{c} & \text { and } & f \nabla^{*} b=\left(f^{c} \triangle^{*} \breve{b}\right)^{c}
\end{array}
$$




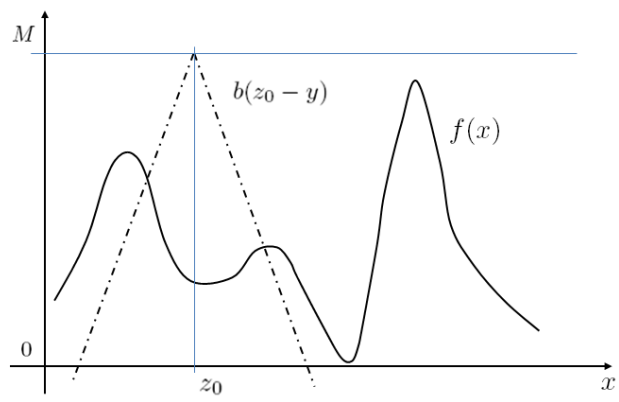

(a)

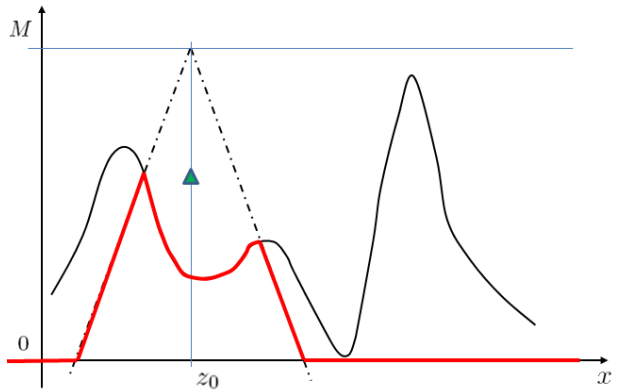

(b)

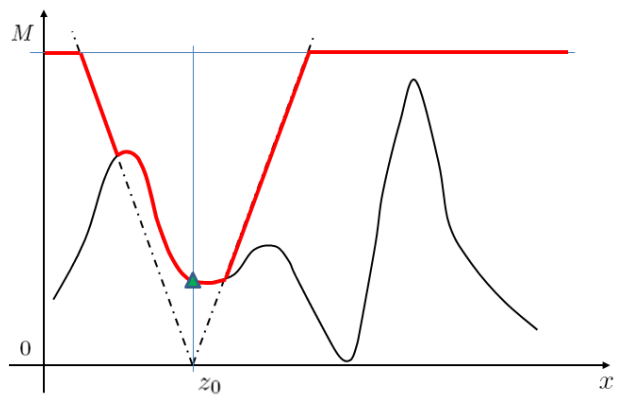

(d)

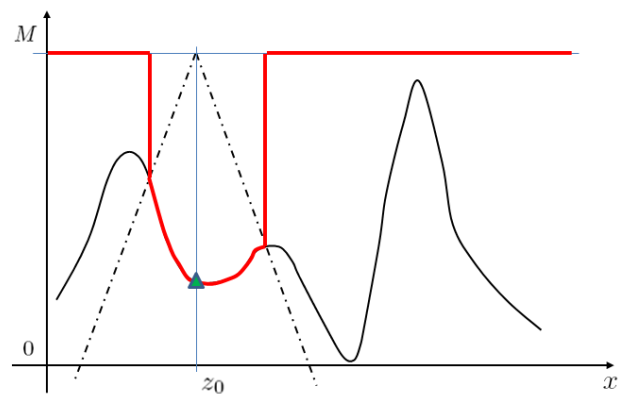

(c)

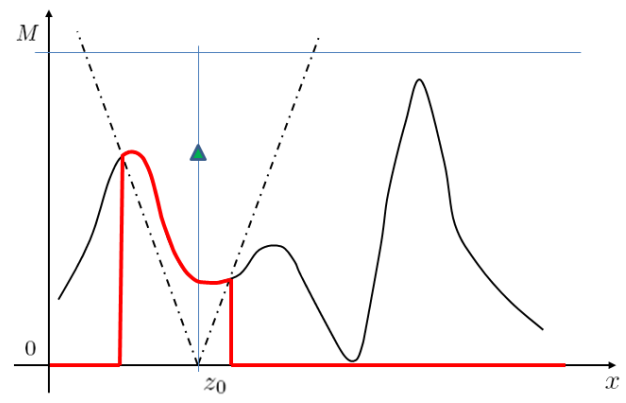

(e)

Figure 1: Illustration of four ( $\max , \min$ )-convolutions for a given example of one dimensional functions defined in a bounded interval, i.e., $f, b \in \mathcal{F}(\mathbb{R},[0, M])$ : (a) original function $f(x)$ and translated structuring function $b$ at point $z_{0}$; (b) in red, $f(y) \wedge b\left(z_{0}-y\right)$ for all $y \in \mathbb{R}$, green triangle represents $(f \nabla b)(x)$ the value of the supmin convolution at $z_{0} ;(\mathrm{c})$ in red, $f(y) \wedge^{*} b\left(y-z_{0}\right)$, green triangle, adjoint infmax at $z_{0}:\left(f \triangle^{*} b\right)\left(z_{0}\right) ;(\mathrm{d})$ in red, $f(y) \vee b^{c}\left(y-z_{0}\right)$, green triangle, infmax at $z_{0}:(f \triangle b)\left(z_{0}\right)$; (e) in red, $f(y) \vee^{*} b^{c}\left(z_{0}-y\right)$, green triangle, adjoint supmin at $z_{0}:\left(f \nabla^{*} b\right)\left(z_{0}\right)$. 
The pair $\left(\triangle^{*}, \nabla\right)$ forms an adjunction. Similarly, the pair $\left(\triangle, \nabla^{*}\right)$ is also an adjunction, i.e., for $f, g, b \in \mathcal{F}\left(\mathbb{R}^{n}, \overline{\mathbb{R}}\right)$ one has

$$
\begin{aligned}
f \nabla b \leq g & \Longleftrightarrow f \leq g \triangle^{*} b \\
f \nabla^{*} b \leq g & \Longleftrightarrow f \leq g \triangle b
\end{aligned}
$$

These relationships are summarized in the following intertwining diagram:

$$
\begin{array}{cll}
(f \nabla b) & \stackrel{\text { adjoint }}{\longleftrightarrow} & \left(f \triangle^{*} b\right) \\
\uparrow \text { dual } & & \uparrow \text { dual } \\
(f \triangle b) & \stackrel{\text { adjoint }}{\longleftrightarrow} & \left(f \nabla^{*} b\right)
\end{array}
$$

Proof. Let us derive the adjunction relationship (28) of pair $\left(\triangle^{*}, \nabla\right)$. First of all, we need to obtain the adjoint operation to the min of two values in a general complete lattice $\mathcal{L}$, whose extreme elements are $\top$ and $\perp$. More precisely, given any triplet $\alpha, t, s \in \mathcal{L}$, as discussed in Section 2, the adjunction involves

$$
t \wedge \alpha \leq s \Leftrightarrow t \leq s \wedge^{*} \alpha
$$

which gives us the corresponding adjoint $\wedge^{*}$ :

$$
s \wedge^{*} \alpha=\sup \{u \in \mathcal{L}: u \wedge \alpha \leq s\}=\left\{\begin{array}{cc}
s & \text { if } \alpha>s \\
\top & \text { if } \alpha \leq s
\end{array}\right.
$$

Now, for any $f, g, b \in \mathcal{F}\left(\mathbb{R}^{n}, \overline{\mathbb{R}}\right)$

$$
\begin{aligned}
f \nabla b \leq g & \Leftrightarrow \forall x \in \mathbb{R}^{n},(f \nabla b)(x) \leq g(x), \\
& \Leftrightarrow \forall x \in \mathbb{R}^{n}, \sup _{y \in \mathbb{R}^{n}}\{f(y) \wedge b(x-y)\} \leq g(x), \\
& \Leftrightarrow \forall x \in \mathbb{R}^{n}, \forall y \in \mathbb{R}^{n}, f(y) \wedge b(x-y) \leq g(x), \\
& \Leftrightarrow \forall x \in \mathbb{R}^{n}, \forall y \in \mathbb{R}^{n}, f(y) \leq g(x) \wedge^{*} b(x-y), \\
& \Leftrightarrow \forall y \in \mathbb{R}^{n}, f(y) \leq \inf _{x \in \mathbb{R}^{n}}\left\{g(x) \wedge^{*} b(x-y)\right\}=\left(g \triangle^{*} b\right)(y), \\
& \Leftrightarrow f \leq g \triangle^{*} b .
\end{aligned}
$$

The second adjunction can be proved analogously, once the adjoint $\mathrm{V}^{*}$ is obtained. We have now

$$
t \vee^{*} \alpha \leq s \Leftrightarrow t \leq s \vee \alpha
$$

such that

$$
t \vee^{*} \alpha=\inf \{u \in \mathcal{L}: t \leq u \vee \alpha\}= \begin{cases}t & \text { if } \alpha<t \\ \perp & \text { if } \alpha \geq t\end{cases}
$$


The duality of $\nabla$ and $\triangle$ is easily proven

$$
\begin{aligned}
\left(f^{c} \nabla \check{b}\right)^{c} & =\left[\sup _{y}\left\{f^{c}(y) \wedge \check{b}(x-y)\right\}\right]^{c}=\inf _{y}\left\{\left[f^{c}(y) \wedge b(y-x)\right]^{c}\right\} \\
& =\inf _{y}\left\{f(y) \vee b^{c}(y-x)\right\}=f \triangle b .
\end{aligned}
$$

Similarly, for the duality between $\nabla^{*}$ and $\triangle^{*}$ it is simply required that

$$
\left[f^{c}(y) \wedge^{*} \check{b}(y-x)\right]^{c}=f(y) \vee^{*} b^{c}(x-y) .
$$

We find

$$
\left[f^{c}(y) \wedge^{*} \check{b}(y-x)\right]^{c}=\left[\left\{\begin{array}{cll}
f^{c}(y) & \text { if } b(x-y)>f^{c}(y) \\
\top & \text { if } b(x-y) \leq f^{c}(y)
\end{array}\right]^{c}=\left\{\begin{array}{cll}
f(y) & \text { if } & b^{c}(x-y)<f(y) \\
\perp & \text { if } & b^{c}(x-y) \geq f(y)
\end{array}\right.\right.
$$

\subsection{Commutation with level set processing}

We can introduce now the fundamental property of (max, $\min$ )-convolutions. We adopt here the convention

$$
X \oplus \emptyset=\emptyset \oplus X=\emptyset .
$$

Proposition 3 Let $f$ and $b$ in $\mathcal{F}\left(\mathbb{R}^{n}, \overline{\mathbb{R}}\right)$. Then the four (max, min)-convolutions of $f$ by $b$ obey the following commutation rules of level sets with respect to Minkowski sum and substraction: for all $h \in \overline{\mathbb{R}}$

$$
\begin{aligned}
X_{h}^{+}(f \nabla b) & =X_{h}^{+}(f) \oplus X_{h}^{+}(b) \\
Y_{h}^{-}(f \triangle b) & =Y_{h}^{-}(f) \oplus Y_{h}^{-}\left(\check{b}^{c}\right) \\
X_{h}^{+}\left(f \triangle^{*} b\right) & =X_{h}^{+}(f) \ominus X_{h}^{+}(b) \\
Y_{h}^{-}\left(f \nabla^{*} b\right) & =Y_{h}^{-}(f) \ominus Y_{h}^{-}\left(\check{b}^{c}\right)
\end{aligned}
$$

Proof. Let us consider the expression of the supmin convolution (20)

$$
X_{h}^{+}(f \nabla b)(x)=\left\{x \in \mathbb{R}^{n} ; \bigvee_{y \in \mathbb{R}^{n}} f(y) \wedge b(x-y) \geq h\right\}
$$

Identifying with terms in equation (10), we can write

$$
X_{h}^{+}(f \nabla b)(x)=\left\{x \in \mathbb{R}^{n} ; \exists y:[f(y) \wedge b(x-y)] \geq h\right\} .
$$

The expression of $X_{h}^{+}(f \nabla b)$ as function of the $X_{h}^{+}(f)$ and $X_{h}^{+}(b)$ comes from the relationship (11), which gives

$$
\left\{x \in \mathbb{R}^{n}: X_{h}^{+}(f) \cap X_{h}^{+}\left(\check{b}_{x}\right) \neq \emptyset\right\}=\left\{x \in \mathbb{R}^{n}: X_{h}^{+}(f) \cap \check{X}_{h}^{+}\left(b_{x}\right) \neq \emptyset\right\},
$$


finally from Minkowski addition (2), we obtain

$$
X_{h}^{+}(f \nabla b)=X_{h}^{+}(f) \oplus X_{h}^{+}(b) .
$$

Now for the infmax convolution $(f \triangle b)$, from expression (13), we have

$$
\begin{aligned}
Y_{h}^{-}(f \triangle b)(x) & =\left\{x \in \mathbb{R}^{n} ; \bigwedge_{y \in \mathbb{R}^{n}} f(y) \vee b^{c}(y-x)<h\right\} \\
& =\left\{x \in \mathbb{R}^{n} ; \exists y:\left[f(y) \vee \check{b}^{c}(x-y)\right]<h\right\},
\end{aligned}
$$

next, using (12), we get

$$
\left\{x \in \mathbb{R}^{n}: Y_{h}^{-}(f) \cap Y_{h}^{-}\left(b_{x}^{c}\right) \neq \emptyset\right\}=\left\{x \in \mathbb{R}^{n}: Y_{h}^{-}(f) \cap \check{Y}_{h}^{-}\left(\check{b}_{x}^{c}\right) \neq \emptyset\right\},
$$

SO

$$
Y_{h}^{-}(f \triangle b)=Y_{h}^{-}(f) \oplus Y_{h}^{-}\left(\check{b}^{c}\right) .
$$

The expression for the adjoint infmax $\left(f \triangle^{*} b\right)$ can be obtained just by adjunction:

$$
\begin{aligned}
\sup \left\{h \in \overline{\mathbb{R}}: x \in X_{h}^{+}(f \nabla b)\right\} \leq g(x) & \Leftrightarrow \cup\left\{k \geq h: X_{k}^{+}(f \nabla b)\right\} \subseteq X_{h}^{+}(g) \forall h \in \overline{\mathbb{R}}, \\
& \Leftrightarrow X_{k}^{+}(f \nabla b) \subseteq X_{h}^{+}(g), \forall h \in \overline{\mathbb{R}}, \forall k \geq h, \\
& \Leftrightarrow X_{k}^{+}(f) \oplus X_{k}^{+}(b) \subseteq X_{h}^{+}(g), \forall h \in \overline{\mathbb{R}}, \forall k \geq h, \\
& \Leftrightarrow X_{k}^{+}(f) \subseteq X_{h}^{+}(g) \ominus X_{k}^{+}(b), \forall h \in \overline{\mathbb{R}}, \forall k \geq h, \\
& \Leftrightarrow X_{k}^{+}(f) \subseteq X_{h}^{+}(g) \ominus X_{k}^{+}(b), \forall k \in \overline{\mathbb{R}}, \forall h \leq k, \\
& \Leftrightarrow X_{k}^{+}(f) \subseteq \cap\left\{h \leq k: X_{h}^{+}(g) \ominus X_{k}^{+}(b)\right\}, \forall k \in \overline{\mathbb{R}}, \\
& \Leftrightarrow X_{k}^{+}(f) \subseteq \cap\left\{h \leq k: X_{h}^{+}(g)\right\} \ominus X_{k}^{+}(b), \forall k \in \overline{\mathbb{R}}, \\
& \Leftrightarrow X_{k}^{+}(f) \subseteq X_{k}^{+}(g) \ominus X_{k}^{+}(b), \forall k \in \overline{\mathbb{R}}, \\
& \Leftrightarrow f(x) \leq \sup \left\{k \in \overline{\mathbb{R}}: x \in\left(X_{k}^{+}(g) \ominus X_{k}^{+}(b)\right)\right\},
\end{aligned}
$$

and consequently, we have

$$
X_{k}^{+}\left(g \triangle^{*} b\right)=X_{k}^{+}(g) \ominus X_{k}^{+}(b) .
$$

By a similar mechanism of adjunction, the expression for the adjoint supmin $\left(f \nabla^{*} b\right)$ is obtained from $Y_{h}^{-}(f \triangle b)=Y_{h}^{-}(f) \oplus Y_{h}^{-}\left(\check{b}^{c}\right)$.

By the way, using the equivalence $X_{h}^{+}\left(f^{c}\right)=X_{-h}^{-}(f)$, we formulate the level set representation for the infmax convolution by duality by complement from the supmin convolution, we obtain

$$
\begin{aligned}
\left(f^{c} \nabla \check{b}\right)^{c} & =\left[\sup \left\{h \in \mathbb{R}: X_{h}^{+}\left(f^{c}\right) \oplus X_{h}^{+}(\check{b})\right\}\right]^{c}=\inf \left\{-h \in \mathbb{R}: X_{h}^{+}\left(f^{c}\right) \oplus X_{h}^{+}(\check{b})\right\} \\
& =\inf \left\{-h \in \mathbb{R}: X_{-h}^{-}(f) \oplus X_{h}^{+}(\check{b})\right\}=\inf \left\{h \in \mathbb{R}: X_{h}^{-}(f) \oplus X_{-h}^{+}(\check{b})\right\} \\
& =\inf \left\{h \in \mathbb{R}: X_{h}^{-}(f) \oplus X_{h}^{-}\left(\check{b}^{c}\right)\right\}=(f \triangle b) .
\end{aligned}
$$


This expression on strict lower level sets $Y_{h}^{-}$for $(f \triangle b)$ is valid for lower level sets $X_{h}^{-}$if ( $f \triangle b$ ) is exact, in the sense that, for each $x \in \operatorname{dom}^{-}(f \triangle b)$, there exists $y \in \mathbb{R}^{n}$ such that $(f \triangle b)(x)=f(y) \vee b^{c}(y-x)$ (i.e., the minimum is attained for any $x$ in the domain) [57, 39]. In particular, if $f$ and $b^{c}$ are both LSC quasiconvex functions, $(f \triangle b)$ and $\left(f \nabla^{*} b\right)$ are exact, which involves:

$$
\begin{aligned}
X_{h}^{-}(f \triangle b) & =X_{h}^{-}(f) \oplus X_{h}^{-}\left(\check{b}^{c}\right) \\
X_{h}^{-}\left(f \nabla^{*} b\right) & =X_{h}^{-}(f) \ominus X_{h}^{-}\left(\check{b}^{c}\right) .
\end{aligned}
$$

We need for the sequel an alternative formulation of the infmax and adjoint supmin convolution in terms respectively of Minkowski subtraction $\ominus$ and addition $\oplus$ of level sets. It is simply based on rewritten the infmax convolution using upper level sets:

$$
\begin{aligned}
(f \triangle b)(x) & =\inf \left\{h \in \mathbb{R}: x \in Y_{h}^{-}(f \triangle b)\right\} \\
& =\sup \left\{h \in \mathbb{R}: x \notin Y_{h}^{-}(f \triangle b)\right\} \\
& =\sup \left\{h \in \mathbb{R}: x \in\left[Y_{h}^{-}(f \triangle b)\right]^{c}\right\} \\
& =\sup \left\{h \in \mathbb{R}: x \in\left[Y_{h}^{-}(f) \oplus Y_{h}^{-}\left(\check{b}^{c}\right)\right]^{c}\right\} \\
& =\sup \left\{h \in \mathbb{R}: x \in X_{h}^{+}(f) \ominus \check{Y}_{h}^{-}\left(\check{b}^{c}\right)\right\} \\
& =\sup \left\{h \in \mathbb{R}: x \in\left(X_{h}^{+}(f) \ominus Y_{h}^{-}\left(b^{c}\right)\right)\right\} .
\end{aligned}
$$

Analogously, one obtain the following equivalence for the adjoint supmin convolution:

$$
\begin{aligned}
\left(f \nabla^{*} b\right)(x) & =\inf \left\{h \in \mathbb{R}: x \in\left(Y_{h}^{-}(f) \ominus Y_{h}^{-}\left(\check{b}^{c}\right)\right)\right\} \\
& =\sup \left\{h \in \mathbb{R}: x \in\left(X_{h}^{+}(f) \oplus Y_{h}^{-}\left(b^{c}\right)\right)\right\} .
\end{aligned}
$$

Therefore, we can write

$$
\begin{aligned}
X_{h}^{+}(f \triangle b) & =X_{h}^{+}(f) \ominus Y_{h}^{-}\left(b^{c}\right), \\
X_{h}^{+}\left(f \nabla^{*} b\right) & =X_{h}^{+}(f) \oplus Y_{h}^{-}\left(b^{c}\right) .
\end{aligned}
$$

As an immediate consequence of Proposition 3, one gets

$$
\begin{aligned}
\operatorname{dom}^{+}(f \nabla b) & =\operatorname{dom}^{+}(f) \oplus \operatorname{dom}^{+}(b), \\
\operatorname{dom}^{-}(f \triangle b) & =\operatorname{dom}^{-}(f) \oplus \operatorname{dom}^{-}(b),
\end{aligned}
$$

where

$$
\begin{aligned}
& \operatorname{dom}^{+}(\phi)=\left\{x \in \mathbb{R}^{n}: \phi(x)>-\infty\right\}, \\
& \operatorname{dom}^{-}(\phi)=\left\{x \in \mathbb{R}^{n}: \phi(x)<+\infty\right\},
\end{aligned}
$$


stand for the effective domains of $\phi \in \mathcal{F}\left(\mathbb{R}^{n}, \overline{\mathbb{R}}\right)$.

Remark. We should clarify that the interest of these expressions of (max, min)-convolutions in terms of Minkowski addition and subtraction is not to suggest an implementation by level set processing followed by stacking. On the contrary, the idea is to show how some morphological operators formulated by level set transforms have a straightforward implementation using (max, min)-convolutions. By the way, the results that we provided are extremely useful to state other properties.

Bibliographic remark. Classically in convex analysis literature [57], definition of level set addition of $f$ by $g$ is given by $(f \triangle g)(x)=\inf _{y}\{f(x-y) \vee g(y)\}$ and its symmetric operation as $(f \nabla g)(x)=\sup _{y}\{f(x-y) \wedge g(y)\}$ such that $(f \nabla g)=-((-f) \nabla(-g))$. This was historically motivated by the fact that the level sum property was pioneered by Rockafellar [55] as $\{f \triangle g<\alpha\}=\{f<\alpha\}+\{g<\alpha\}, \forall \alpha \in \overline{\mathbb{R}}$, where the notation $\{h<\alpha\}=\left\{x \in \mathbb{R}^{n}: h(x)<\alpha\right\}$ is used for the strict lower level set of $h$ and + refers in this context to Minkowski addition.

\subsection{Further properties}

Proposition 4 We have the following properties for the (max, min)-convolutions.

1. (Increaseness) If $f(x) \leq g(x), \forall x \in \mathbb{R}^{n}, f, g \in \mathcal{F}\left(\mathbb{R}^{n}, \overline{\mathbb{R}}\right)$, then $(f \nabla b)(x) \leq(g \nabla b)(x)$, $(f \triangle b)(x) \leq(g \triangle b)(x),\left(f \nabla^{*} b\right)(x) \leq\left(g \nabla^{*} b\right)(x)$ and $\left(f \triangle^{*} b\right)(x) \leq\left(g \triangle^{*} b\right)(x), \forall x \in \mathbb{R}^{n}$ and for any $b \in \mathcal{F}(E, \overline{\mathbb{R}})$.

2. (Extreme values preservation) Given any function $f \in \mathcal{F}\left(\mathbb{R}^{n}, \overline{\mathbb{R}}\right)$ and structuring function $b \in \mathcal{F}\left(\mathbb{R}^{n}, \overline{\mathbb{R}}\right)$, one has

$$
\begin{aligned}
\max (f \nabla b) & =(\max f) \wedge(\max b) \\
\min (f \triangle b) & =(\min f) \vee\left(\min b^{c}\right)=(\min f) \vee(\max b), \\
\max \left(f \nabla^{*} b\right) & =\max f \\
\min \left(f \triangle^{*} b\right) & =\min f .
\end{aligned}
$$

3. (Distributivity - Commutation with supremum and infimum) Given a structuring function $b \in \mathcal{F}\left(\mathbb{R}^{n}, \overline{\mathbb{R}}\right)$ and an arbitrary family $\left\{f_{i}\right\}, i \in I, f_{i} \in \mathcal{F}\left(\mathbb{R}^{n}, \overline{\mathbb{R}}\right)$, it follows $\forall x \in E$ that

$$
\begin{gathered}
\left(\sup _{i \in I} f_{i} \nabla b\right)(x)=\sup _{i \in I}\left(f_{i} \nabla b\right)(x) \quad ; \quad\left(\inf _{i \in I} f_{i} \triangle b\right)(x)=\inf _{i \in I}\left(f_{i} \triangle b\right)(x) \\
\left(\sup _{i \in I} f_{i} \nabla^{*} b\right)(x)=\sup _{i \in I}\left(f_{i} \nabla^{*} b\right)(x) \quad ; \quad\left(\inf _{i \in I} f_{i} \triangle^{*} b\right)(x)=\inf _{i \in I}\left(f_{i} \triangle^{*} b\right)(x)
\end{gathered}
$$


4. (Associativity - Combination of several structuring functions) Given any function $f \in$ $\mathcal{F}\left(\mathbb{R}^{n}, \overline{\mathbb{R}}\right)$ and any pair of structuring functions $b_{1}, b_{2} \in \mathcal{F}\left(\mathbb{R}^{n}, \overline{\mathbb{R}}\right)$, one obtains

$$
\begin{aligned}
\left(f \nabla b_{1}\right) \nabla b_{2} & =f \nabla\left(b_{1} \nabla b_{2}\right)=f \nabla\left(b_{2} \nabla b_{1}\right), \\
\left(f \triangle b_{1}\right) \triangle b_{2} & =f \triangle\left(b_{1} \nabla b_{2}\right)=f \triangle\left(b_{2} \nabla b_{1}\right), \\
\left(f \triangle^{*} b_{1}\right) \triangle^{*} b_{2} & =f \triangle^{*}\left(b_{1} \nabla b_{2}\right)=f \triangle^{*}\left(b_{2} \nabla b_{1}\right), \\
\left(f \nabla^{*} b_{1}\right) \nabla^{*} b_{2} & =f \nabla^{*}\left(b_{1} \nabla b_{2}\right)=f \nabla^{*}\left(b_{2} \nabla b_{1}\right) .
\end{aligned}
$$

Proof. The increaseness is straightforward since all the operations involved in the (max, min)convolutions for a fixed $b$ are increasing.

The proof for the extreme value preservation of supmin convolution is as follows:

$$
\begin{aligned}
\max (f \nabla b)(x) & =\sup _{x \in \mathbb{R}^{n}} \sup _{y, z \in \mathbb{R}^{n}}\{f(y) \wedge b(z), y+z=x\} \\
& =\sup _{y, z \in \mathbb{R}^{n}}\{f(y) \wedge b(z)\}=\left(\sup _{y \in \mathbb{R}^{n}} f(y)\right) \wedge\left(\sup _{z \in \mathbb{R}^{n}} b(z)\right) .
\end{aligned}
$$

Similarly for $\min (f \triangle b)$. For the adjoint supmin convolution, we have:

$$
\begin{aligned}
& \max \left(f \nabla^{*} b\right)(x)=\sup _{x \in \mathbb{R}^{n}} \sup _{y, z \in \mathbb{R}^{n}}\left\{\begin{array}{cll}
f(y) & \text { if } b^{c}(z)<f(y) \\
\perp & \text { if } b^{c}(z) \geq f(y)
\end{array}=\right. \\
& \sup _{y, z \in \mathbb{R}^{n}}\left\{\begin{array}{cll}
f(y) & \text { if } b^{c}(z)<f(y) \\
\perp & \text { if } b^{c}(z) \geq f(y)
\end{array}=\left(\sup _{y, z \in \mathbb{R}^{n}, b^{c}(z)<f(y)} f(y)\right) \vee\left(\sup _{y, z \in \mathbb{R}^{n}, b^{c}(z) \geq f(y)} \perp\right)=\max f .\right.
\end{aligned}
$$

Analogously for $\max \left(f \triangle^{*} b\right)$.

We prove the distributivity of supmin convolution and the adjoint supmin convolution. For the two other operators the procedure is similar. One has for the supmin convolution of a sup of functions:

$$
\begin{aligned}
\left(\sup _{i \in I} f_{i} \nabla b\right)(x) & =\sup _{y \in \mathbb{R}^{n}}\left\{\sup _{i \in I} f_{i}(y) \wedge b(x-y)\right\} \\
& =\sup _{i \in I} \sup _{y \in \mathbb{R}^{n}}\left\{f_{i}(y) \wedge b(x-y)\right\}=\left(f_{i} \nabla b\right)(x) .
\end{aligned}
$$

In the case of the adjoint supmin convolution of the sup of function:

$$
\left(\sup _{i \in I} f_{i} \nabla^{*} b\right)(x)=\sup _{y \in \mathbb{R}^{n}}\left\{\begin{array}{cll}
\sup _{i \in I} f_{i}(y) & \text { if } & b^{c}(x-y)<\sup _{i \in I} f_{i}(y) \\
\perp & \text { if } & b^{c}(x-y) \geq \sup _{i \in I} f_{i}(y)
\end{array}\right.
$$


We note the the cases where $b^{c}(x-y) \geq \sup _{i \in I} f_{i}(y)$ involves $b^{c}(x-y) \geq f_{i}(y), \forall i \in I$. Therefore we can write

$$
\left(\sup _{i \in I} f_{i} \nabla^{*} b\right)(x)=\sup _{i \in I} \sup _{y \in \mathbb{R}^{n}}\left\{\begin{array}{cll}
f_{i}(y) & \text { if } b^{c}(x-y)<f_{i}(y) \\
\perp & \text { if } \quad b^{c}(x-y) \geq f_{i}(y)
\end{array}=\sup _{i \in I}\left(f_{i} \nabla^{*} b\right)(x) .\right.
$$

The associativity of structuring functions is easily obtained using the level set expressions and the following classical results [46]

$$
\left(X \oplus B_{1}\right) \oplus B_{2}=X \oplus\left(B_{1} \oplus B_{2}\right) ; \quad\left(X \ominus B_{1}\right) \ominus B_{2}=X \ominus\left(B_{1} \oplus B_{2}\right) .
$$

Thus, we have:

$$
\begin{aligned}
X_{h}^{+}\left(\left(f \nabla b_{1}\right) \nabla b_{2}\right) & =X_{h}^{+}\left(f \nabla b_{1}\right) \oplus X_{h}^{+}\left(b_{2}\right)=X_{h}^{+}(f) \oplus X_{h}^{+}\left(b_{1}\right) \oplus X_{h}^{+}\left(b_{2}\right) \\
& =X_{h}^{+}(f) \oplus X_{h}^{+}\left(b_{1} \nabla b_{2}\right) . \\
Y_{h}^{-}\left(\left(f \triangle b_{1}\right) \triangle b_{2}\right) & =Y_{h}^{-}\left(f \triangle \check{b}_{1}^{c}\right) \oplus Y_{h}^{-}\left(\check{b}_{2}^{c}\right)=Y_{h}^{-}(f) \oplus Y_{h}^{-}\left(\check{b}_{1}^{c}\right) \oplus Y_{h}^{-}\left(\check{b}_{2}^{c}\right) \\
& =Y_{h}^{-}(f) \oplus\left(Y_{-h}^{+}\left(\check{b}_{1}\right) \oplus Y_{-}^{+}\left(\check{b}_{2}\right)\right)=Y_{h}^{-}(f) \oplus \check{Y}_{-h}^{+}\left(b_{1} \nabla b_{2}\right) \\
& =Y_{h}^{-}(f) \oplus \check{Y}_{h}^{-}\left(\left(b_{1} \nabla b_{2}\right)^{c}\right) . \\
X_{h}^{+}\left(\left(f \triangle^{*} b_{1}\right) \triangle^{*} b_{2}\right) & =X_{h}^{+}\left(f \triangle^{*} b_{1}\right) \ominus X_{h}^{+}\left(b_{2}\right)=\left[X_{h}^{+}(f) \ominus X_{h}^{+}\left(b_{1}\right)\right] \ominus X_{h}^{+}\left(b_{2}\right) \\
& =X_{h}^{+}(f) \ominus\left(X_{h}^{+}\left(b_{1}\right) \oplus X_{h}^{+}\left(b_{2}\right)\right)=X_{h}^{+}(f) \ominus X_{h}^{+}\left(b_{1} \nabla b_{2}\right) . \\
Y_{h}^{-}\left(\left(f \nabla^{*} b_{1}\right) \nabla^{*} b_{2}\right)= & Y_{h}^{-}\left(f \nabla^{*} b_{1}\right) \ominus Y_{h}^{-}\left(\check{b}_{2}^{c}\right)=\left[Y_{h}^{-}(f) \ominus Y_{h}^{-}\left(\check{b}_{1}^{c}\right)\right] \ominus Y_{h}^{-}\left(\check{b}_{2}^{c}\right) \\
= & Y_{h}^{-}(f) \ominus\left(Y_{h}^{-}\left(\check{b}_{1}^{c}\right) \oplus Y_{h}^{-}\left(\check{b}_{2}^{c}\right)\right)=Y_{h}^{-}(f) \ominus\left(\check{Y}_{-h}^{+}\left(b_{1}\right) \oplus \check{Y}_{-h}^{+}\left(b_{2}\right)\right) \\
= & Y_{h}^{-}(f) \ominus \check{Y}_{-h}^{+}\left(b_{1} \nabla b_{2}\right)=Y_{h}^{-}(f) \ominus \check{Y}_{h}^{-}\left(\left(b_{1} \nabla b_{2}\right)^{c}\right) .
\end{aligned}
$$

Canonic structuring function. The conic structuring function plays a role similar to the multiscale quadratic structuring function $[22,41,30]$ in $(\max ,+)$-algebra.

Definition 5 The multiscale conic structuring function is defined as the canonic structuring function in ( $\max , \min )$-convolutions:

$$
c_{\lambda}(x)=-\frac{\|x\|}{\lambda}
$$

In order to justify this canonicity, let us consider the upper level sets of $c_{\lambda}(x)$. First, we remind that a ball of radius centered at point $x$ is given by the set

$$
B_{r}(x)=\left\{y \in \mathbb{R}^{n}:\|x-y\| \leq r\right\} .
$$


Proposition 6 The canonic structuring function in (max, min)-convolutions satisfies the semi-group

$$
\left(c_{\lambda} \nabla c_{\mu}\right)(x)=c_{\lambda+\mu}(x) .
$$

In the case of the $L^{\infty}$ metric, a dimension separability is obtained for $c_{\lambda}^{\infty}(x)=-\|x\|_{\infty} / \lambda$; i.e., let us denote the coordinates of point as $x=\left(x_{1}, x_{2}, \cdots, x_{n}\right)$ and by $c_{\lambda ; i}(x)=-\left|x_{i}\right| / \lambda$ the one dimensional conic structuring function, we have

$$
c_{\lambda}^{\infty}(x)=\left(c_{\lambda ; 1} \nabla c_{\lambda ; 2} \cdots \nabla c_{\lambda ; n}\right)
$$

Proof. We first note that $X_{-h}^{+}\left(c_{\lambda}\right)=B_{\lambda h}$. Second, we remind the Minkowski addition of balls: $B_{r_{1}} \oplus B_{r_{2}}=B_{r_{1}+r_{2}}$. Therefore, one has

$$
X_{-h}^{+}\left(c_{\lambda} \nabla c_{\mu}\right)=X_{-h}^{+}\left(c_{\lambda}\right) \oplus X_{-h}^{+}\left(c_{\mu}\right)=B_{\lambda h} \oplus B_{\mu h}=B_{(\lambda+\mu) h}
$$

Dimension separability in $L^{\infty}$ metric is also a consequence of the Minkowski addition of segments.

As a consequence of the $L^{\infty}$ dimension separability, the classical theory of Minkowski decomposition of structuring elements [58], e.g., approximate isotropic structuring elements such as octagons in the square grid or dodecagons in the hexagonal grid using one dimensional structuring elements, can be extended to the case of functions in ( $\max , \min$ )-convolutions.

Concerning this point, we note that the dimension separability of the Euclidean quadratic structuring in the $(\max ,+)$-convolutions is a richer property leading to efficient decompositions of separable concave structuring functions [23].

\subsection{Openings, closings using (max, min)-convolutions and granulometries}

The adjointness of the pairs $\left(\triangle^{*}, \nabla\right)$ and $\left(\triangle, \nabla^{*}\right)$ involves that from an algebraic viewpoint both the supmin convolution $\nabla$ and the adjoint supmin convolution $\nabla^{*}$ are a dilation; both the infmax convolution $\triangle$ and the adjoint infmax convolution $\triangle^{*}$ are an erosion. Therefore, their composition naturally yields openings and closings. Let us be more precise.

Definition 7 Given any USC function $f \in \mathcal{F}\left(\mathbb{R}^{n}, \overline{\mathbb{R}}\right)$, the (max, min)-opening and (max, min)closing of $f$ by the continuous structuring function $b \in \mathcal{F}\left(\mathbb{R}^{n}, \overline{\mathbb{R}}\right)$ are respectively given by

$$
(f \diamond b)=\left(\left(f \triangle^{*} b\right) \nabla b\right),
$$

and

$$
(f \triangleleft b)=\left(\left(f \nabla^{*} b\right) \triangle b\right),
$$


such that their corresponding level sets representations, based on expressions (30), (32), and (31), (33), are given by

$$
\begin{aligned}
X_{h}^{+}(f \diamond b) & =X_{h}^{+}\left(f \triangle^{*} b\right) \oplus X_{h}^{+}(b)=\left[X_{h}^{+}(f) \ominus X_{h}^{+}(b)\right] \oplus X_{h}^{+}(b) \\
& =X_{h}^{+}(f) \circ X_{h}^{+}(b), \\
Y_{h}^{-}(f \diamond b) & =Y_{h}^{-}\left(f \nabla^{*} b\right) \oplus Y_{h}^{-}\left(\check{b}^{c}\right)=\left[Y_{h}^{-}(f) \ominus Y_{h}^{-}\left(\check{b}^{c}\right)\right] \oplus Y_{h}^{-}\left(\check{b}^{c}\right) \\
& =Y_{h}^{-}(f) \circ Y_{h}^{-}\left(\check{b}^{c}\right) .
\end{aligned}
$$

We note that (max, min)-opening is defined from adjunction $\left(\triangle^{*}, \nabla\right)$ whereas $(\max , \min )$ closing from $\left(\triangle, \nabla^{*}\right)$. We can also switch roles and to formulate the so-called second family of dual (max, min)-opening and closing as

$$
\begin{aligned}
& \left(f \diamond^{*} b\right)=\left((f \triangle b) \nabla^{*} b\right), \\
& \left(f \triangleright^{*} b\right)=\left((f \nabla b) \triangle^{*} b\right),
\end{aligned}
$$

which has the following equivalent interpretation in terms of level sets:

$$
\begin{aligned}
Y_{h}^{-}\left(f \diamond^{*} b\right) & =Y_{h}^{-}(f) \bullet Y_{h}^{-}\left(\check{b}^{c}\right), \\
X_{h}^{+}\left(f \diamond^{*} b\right) & =X_{h}^{+}(f) \bullet X_{h}^{+}(b) .
\end{aligned}
$$

Besides the duality by complement, classical properties of opening and closing hold in the (max, min) framework.

Proposition 8 For any $f, b \in \mathcal{F}\left(\mathbb{R}^{n}, \overline{\mathbb{R}}\right)$, we have,

1. $(f \diamond b)$ and $(f \diamond b)$ are dual operators

$$
(f \diamond b)=\left(f^{c} \breve{b}\right)^{c} \quad \text { and } \quad(f \diamond b)=\left(f^{c} \diamond \breve{b}\right)^{c}
$$

2. $(f \diamond b)$ and $(f \diamond b)$ are both increasing operators.

3. $(f \diamond b)$ is anti-extensive and $(f \diamond b)$ extensive with the following ordering relationship:

$$
(f \diamond b)(x) \leq f(x) \leq(f \diamond b)(x), \forall x \in \mathbb{R}^{n} ;
$$

4. idempotency of both operators: $((f \diamond b) \diamond b)=(f \diamond b)$ and $((f \diamond b) \diamond b)=(f \diamond b)$.

Property 1 can be easily proved:

$$
\begin{aligned}
\left(f^{c} b\right) & =\left(\left(f^{c} \nabla^{*} b\right) \triangle b\right)=\left(\left(f \triangle^{*} \check{b}\right)^{c} \triangle b\right)=\left(\left(f \triangle^{*} \check{b}\right) \nabla \check{b}\right)^{c} \\
& =(f \diamond \breve{b})^{c} .
\end{aligned}
$$

The other properties are a consequence of the adjunction [29]. 
We note that the second family of (max, min)-opening and closing, $\left(f \diamond^{*} b\right)$ and $\left(f \diamond^{*} b\right)$, do satisfy the same properties.

Property 4 on idempotency together with the increaseness defines a family of operators called algebraic openings/closings [59, 29], larger than the one associated to the composition of a pair of dilation and erosion (structural openings). One of the most classical results in morphological operators provided us an example of algebraic opening: given a collection of openings $\left\{\gamma_{i}\right\}$, increasing, idempotent and anti-extensive operators for all $i$, their supremum $\sup _{i} \gamma_{i}$ is also an opening [46]. A dual result is obtained for the closing by changing the sup to the inf. The class of openings (resp. closings) is neither closed under infimum (resp. opening) or a generic composition. There is however a semi-group property leading to a scale-space framework for opening/closing operators, known as granulometries. The notion of granulometry in Euclidean morphology is summarized in the following result due to Matheron [46] and Serra [59].

Theorem 9 A parameterized family $\left\{\gamma_{\lambda}\right\}_{\lambda>0}$ of flat openings from $\mathcal{F}(E, \mathcal{T})$ into $\mathcal{F}(E, \mathcal{T})$ is a granulometry (or size distribution) when

$$
\gamma_{\lambda_{1}} \gamma_{\lambda_{2}}=\gamma_{\lambda_{2}} \gamma_{\lambda_{1}}=\gamma_{\sup \left(\lambda_{1}, \lambda_{2}\right)} ; \quad \lambda_{1}, \lambda_{2}>0
$$

Condition (53) is equivalent to both

$$
\begin{aligned}
& \gamma_{\lambda_{1}} \leq \gamma_{\lambda_{2}} ; \quad \lambda_{1} \geq \lambda_{2}>0 \\
& \mathcal{B}_{\lambda_{1}} \subseteq \mathcal{B}_{\lambda_{2}} ; \quad \lambda_{1} \geq \lambda_{2}>0
\end{aligned}
$$

where $\mathcal{B}_{\lambda}$ is the invariance domain of the opening at scale $\lambda$; i.e., the family of structuring elements Bs such that $B=\gamma_{\lambda}(B)$ [59].

By duality, we introduce antisize distributions as the families of closings $\left\{\varphi_{\lambda}\right\}_{\lambda>0}$.

Formula (53) shows how translation invariant flat openings are composed and highlights their semi-group structure. The equivalent condition (54) emphasizes the monotonicity of the granulometry with respect to $\lambda$ : the opening becomes more and more active as $\lambda$ increases. When dealing with Euclidean spaces, [46] introduced the notion of Euclidean granulometry as the size distribution being translationally invariant and compatible with homothetics, i.e.,

$$
\gamma_{\lambda}(f(x))=\lambda \gamma_{1}\left(f\left(\lambda^{-1} x\right)\right), \forall \lambda>0
$$

where $f \in \mathcal{F}(E, \mathcal{T})$ is a Euclidean grey-level image. More precisely, a family of mappings $\gamma_{\lambda}$ is a Euclidean granulometry if and only if there exists a class $\mathcal{B}^{\prime}$ such that

$$
\gamma_{\lambda}(f)=\bigvee_{B \in \mathcal{B}^{\prime}} \bigvee_{\mu \geq \lambda} \gamma_{\mu B}(f)
$$


Then, we note that condition (55) involves that the domain of invariance $\mathcal{B}_{\lambda}$ is equal to $\lambda \mathcal{B}$, where $\mathcal{B}$ is the class closed under union, translation and homothetics $\geq 1$, which is generated by $\mathcal{B}^{\prime}$. If we reduce the class $\mathcal{B}^{\prime}$ to a single element $B$, the associated size distribution becomes

$$
\gamma_{\lambda}(f)=\bigvee_{\mu \geq \lambda} \gamma_{\mu B}(f)
$$

The following key result simplifies the situation. The size distribution by a compact structuring element $B$ is equivalent to $\gamma_{\lambda}(f)=\gamma_{\lambda B}(f)$ if and only if $B$ is convex [46].

The extension of the granulometric theory to non-flat structuring functions in the framework of (max, +)-based morphology was deeply studied in [35]. In particular, it was proven that one can build grey-level Euclidean granulometries with a multiscale structuring function $b_{\lambda}$, i.e., $\gamma_{\lambda}(f)=\left(\left(f \ominus b_{\lambda}\right) \oplus b_{\lambda}\right)$ if and only if structuring function $b_{\lambda}$ has a convex compact domain and is constant there (i.e., flat function). In the terminology of [35], (max, +)granulometries are based on the notion of $T$-openings.

In the case of (max, min)-openings, we can naturally extend Matheron axiomatic of Euclidean granulometries without the flatness limitation. The transposition of general formulation of granulometries given in Theorem 9 involves that, fixing $b_{1}, b_{2} \in \mathcal{F}\left(\mathbb{R}^{n}, \overline{\mathbb{R}}\right)$, we have that

$$
\left(f \diamond b_{1}\right) \leq\left(f \diamond b_{2}\right), \text { for every } f \in \mathcal{F}\left(\mathbb{R}^{n}, \overline{\mathbb{R}}\right)
$$

if and only if $\left(b_{1} \diamond b_{2}\right)=b_{1}$.

We focus on the interesting case of Euclidean granulometries from (max, min)-openings.

Proposition 10 Given a structuring function $b_{1} \in \mathcal{F}\left(\mathbb{R}^{n}, \overline{\mathbb{R}}\right)$ such that all its upper level sets $X_{h}^{+}\left(b_{1}\right)$ are convex sets, the family of multi-scale (max, $\min$ )-openings $\left\{f \diamond b_{\lambda}\right\}_{\lambda \geq 1}$, where the structuring function at scale $\lambda$ is given by

$$
b_{\lambda}(x)=b_{1}\left(\lambda^{-1} x\right),
$$

forms an Euclidean granulometry on any image $f \in \mathcal{F}\left(\mathbb{R}^{n}, \overline{\mathbb{R}}\right)$, i.e.,

$$
\left(f \diamond b_{\lambda}\right)=\lambda \star\left(\left(\lambda^{-1} \star f\right) \diamond b_{1}\right),
$$

which involves compatibility with scaling in the spatial domain, in the sense of Matheron's axiomatic defined as follows

$$
(\lambda \star f)(x)=f\left(\lambda^{-1} x\right), \forall \lambda \geq 1
$$

In addition, we have the following semi-group properties, $\forall \lambda_{1}, \lambda_{2} \geq 1$

$$
\begin{aligned}
b_{\lambda_{1}+\lambda_{2}}(x) & =\left(b_{\lambda_{1}} \nabla b_{\lambda_{2}}\right)(x) \\
\left(\left(f \diamond b_{\lambda_{1}}\right) \diamond b_{\lambda_{2}}\right)(x) & =\left(\left(f \diamond b_{\lambda_{2}}\right) \diamond b_{\lambda_{1}}\right)(x)=\left(f \diamond b_{\sup \left(\lambda_{1}, \lambda_{2}\right)}\right)(x)
\end{aligned}
$$


Proof. We need to prove the expression (56). In terms of levels sets, spatial scaling compatibility involves that we have for every $f \in \mathcal{F}\left(\mathbb{R}^{n}, \overline{\mathbb{R}}\right)$

$$
X_{h}^{+}(\lambda \star f)=\lambda X_{h}^{+}(f), \forall h \in \overline{\mathbb{R}} .
$$

Therefore, we have $X_{h}^{+}\left(b_{\lambda}\right)=\lambda X_{h}^{+}\left(b_{1}\right)$. Convexity of upper level sets of $b_{1}$ involves we have the homothetic compatibility of semi-group (57):

$$
X_{h}^{+}\left(b_{\lambda_{1}} \nabla b_{\lambda_{2}}\right)=\lambda_{1} X_{h}^{+}\left(b_{1}\right) \oplus \lambda_{1} X_{h}^{+}\left(b_{2}\right)=\left(\lambda_{1}+\lambda_{2}\right) X_{h}^{+}\left(b_{1}\right)=X_{h}^{+}\left(b_{\lambda_{1}+\lambda_{2}}\right) .
$$

In addition, one has

$$
X_{h}^{+}(f) \circ X_{h}^{+}\left(b_{\lambda}\right)=X_{h}^{+}(f) \circ \lambda X_{h}^{+}\left(b_{1}\right) .
$$

Therefore, the granulometric semi-group (58) is verified at each upper level set:

$$
\left.\left(X_{h}^{+}(f) \circ X_{h}^{+}\left(b_{\lambda_{1}}\right)\right) \circ X_{h}^{+}\left(b_{\lambda_{2}}\right)=\left(X_{h}^{+}(f) \circ X_{h}^{+}\left(b_{\lambda_{2}}\right)\right) \circ X_{h}^{+}\left(b_{\lambda_{1}}\right)=X_{h}^{+}(f) \circ X_{h}^{+}\left(b_{\sup \left(\lambda_{1}, \lambda_{2}\right.}\right)\right) .
$$

We have already all the elements for (56):

$$
\begin{aligned}
\left(f \diamond b_{\lambda}\right)(x) & =\sup \left\{h \in \mathbb{R}: x \in X_{h}^{+}\left(f \diamond b_{\lambda}\right)\right\} \\
& =\sup \left\{h \in \mathbb{R}: x \in\left[X_{h}^{+}(f) \circ X_{h}^{+}\left(b_{\lambda}\right)\right]\right\} \\
& =\sup \left\{h \in \mathbb{R}: x \in\left[X_{h}^{+}(f) \circ \lambda X_{h}^{+}\left(b_{1}\right)\right]\right\} \\
& =\sup \left\{h \in \mathbb{R}: x \in\left[\lambda X_{h}^{+}\left(\lambda^{-1} \star f\right) \circ \lambda X_{h}^{+}\left(b_{1}\right)\right]\right\} \\
& =\sup \left\{h \in \mathbb{R}: \lambda^{-1} x \in\left[X_{h}^{+}\left(\lambda^{-1} \star f\right) \circ X_{h}^{+}\left(b_{1}\right)\right]\right\} \\
& =\sup \left\{h \in \mathbb{R}: \lambda^{-1} x \in X_{h}^{+}\left(\left(\lambda^{-1} \star f\right) \diamond b_{1}\right)\right\} \\
& =\left(\left(\lambda^{-1} \star f\right) \diamond b_{1}\right)\left(\lambda^{-1} x\right) \\
& =\left[\lambda \star\left(\left(\lambda^{-1} \star f\right) \diamond b_{1}\right)\right](x) .
\end{aligned}
$$

A dual result of anti-granulometry is obtained for ( $\max , \min )$ multiscale closings $\left(f b_{\lambda}\right)$.

A good candidate of multi-scale isotropic structuring function leading to (max, min) granulometries is based on the canonic structuring function (41), as $b_{\lambda}(x)=c_{\lambda}(x)+\alpha$, which is equivalent to

$$
b_{\lambda}(x)=\lambda^{-1} c_{1}(x)+\alpha, \quad \lambda \geq 1, \alpha>0 .
$$

More generally, we can also consider the family of multi-scale functions

$$
b_{\lambda}(x)=-\frac{\|x\|^{P}}{\lambda^{P}}+\alpha, \quad \lambda \geq 1, \alpha>0, P>0 .
$$




\section{Hopf-Lax-Oleinik formulas for Hamilton-Jacobi equation $u_{t} \pm$ $H(u, D u)=0$}

Operators formalized in the previous Section are also related to the viscosity solution of a well known family of nonlinear partial differential equations: the Hamilton-Jacobi equation of the form $u_{t} \pm H(u, D u)=0$. Hamilton-Jacobi equations are of great importance in physics and optimal control [18]. Before developing the connection with the present work, let us review very briefly the link between another form of Hamilton-Jacobi equation, $u_{t} \pm H(D u)=0$, and classical morphological operators viewed as convolutions in $(\max ,+)$-algebra.

\subsection{Morphological PDE for classical dilation and erosion}

Consider the Hamilton-Jacobi equation

$$
u_{t}(x, t) \pm H(x, D u(x, t))=0 \text { in } \mathbb{R}^{n} \times(0,+\infty),
$$

with the initial condition $u(x, 0)=f(x)$ in $\mathbb{R}^{n}$. Such family of equations usually does not admit classic (i.e., everywhere differentiable) solutions but can be studied in the framework of the theory of viscosity solutions [19]. It is well known [10] that if the Hamiltonian has the properties: (i) $H(x, p)=H(p)$ is convex and (ii) $\lim _{|p| \rightarrow+\infty} H(p) /|p|=+\infty$, then the solution of Cauchy problem (59) is given for + and - respectively by the so-called Hopf-Lax-Oleinik formulas:

$$
u(x, t)=\inf _{y \in \mathbb{R}^{n}}\left[f(y)+t H^{*}\left(\frac{x-y}{t}\right)\right],
$$

and

$$
u(x, t)=\sup _{y \in \mathbb{R}^{n}}\left[f(y)-t H^{*}\left(\frac{x-y}{t}\right)\right],
$$

where $H^{*}(q)$ is the Legendre-Fenchel transform of function $H(p)$, i.e.,

$$
H^{*}(q)=\sup _{p \in \mathbb{R}^{n}}\{\langle p, q\rangle-H(p)\}, \quad q \in \mathbb{R}^{n} .
$$

where $\langle p, q\rangle$ denotes the dot product of $p$ and $q$.

PDE (59) plays a central role in continuous morphology [1, 7, 17, 42]. In particular, by taking $H(p)=1 / 2\|p\|^{2}$, such that $H^{*}(q)=1 / 2\|q\|^{2}$, a kind of canonic morphological PDE is formulated

$$
\begin{cases}\frac{\partial u}{\partial t}= \pm \frac{1}{2}\|\nabla u\|^{2}, & x \in E, t>0 \\ u(x, 0)=f(x), & x \in E\end{cases}
$$

such that the corresponding viscosity solutions are given by

$$
\begin{array}{ll}
u(x, t)=\sup _{y \in E}\left\{f(y)-\frac{\|x-y\|^{2}}{2 t}\right\} & (\text { for }+\operatorname{sign}), \\
u(x, t)=\inf _{y \in E}\left\{f(y)+\frac{\|x-y\|^{2}}{2 t}\right\} & (\text { for }- \text { sign }),
\end{array}
$$


which just correspond to a dilation $\left(f \oplus p_{t}\right)(x)$ and an erosion $\left(f \ominus p_{t}\right)(x)$ of image $f(x)$ using the multiscale quadratic (or parabolic) structuring function:

$$
p_{t}(x)=-\frac{\|x\|^{2}}{2 t} .
$$

By the way, due to its properties of semi-group, dimension separability and invariance to transform domain $[41,30,15]$, the structuring function $p_{t}(x)$ can be considered as the canonic one in morphology, playing a similar role to the Gaussian kernel in linear filtering.

Other generalized forms of the Hamilton-Jacobi model (59) cover the flat morphology by disks [42]; i.e., $u_{t}= \pm\|\nabla u\|$, as well as operators with more general $P$-power concave

structuring functions, i.e., $u_{t}= \pm\|\nabla u\|^{P}$. For the application of the latter model to adaptive morphology see [21].

\subsection{Viscosity solution of Hamilton-Jacobi equation with Hamiltonians con- taining $u$ and $D u$}

We study now the Hopf-Lax-Oleinik type formulas for Hamilton-Jacobi PDE of form $u_{t} \pm$ $H(u, D u)=0$ and its links to convolutions in (max, min)-algebra. The theory of this equation was developed by Barron, Jensen and Liu [11, 12]. Other interesting results can be found in paper by Alvarez, Barron and Ishii [2] and the excellent survey paper by Van and Son [65]. The most relevant elements for us can be summarized in the following result.

Proposition 11 Let us consider the two following Cauchy problems (first-order HamiltonJacobi PDEs):

$$
\begin{cases}u_{t}+H_{1}(u, D u)=0, & \text { in }(x, t) \in \mathbb{R}^{n} \times(0, \infty), \\ u(x, 0)=f(x), & \forall x \in \mathbb{R}^{n},\end{cases}
$$

and

$$
\begin{cases}u_{t}+H_{2}(u, D u)=0, & \text { in }(x, t) \in \mathbb{R}^{n} \times(0, \infty), \\ u(x, 0)=g(x), & \forall x \in \mathbb{R}^{n},\end{cases}
$$

where the initial conditions are functions $f, g: \mathbb{R}^{n} \times \mathbb{R}$, such that $f$ is a LSC proper function, bounded from below; and $g$ an USC proper function, bounded from above. The Hamiltonians $H_{1}, H_{2}: \mathbb{R} \times \mathbb{R}^{n} \rightarrow \mathbb{R}^{n}$ are assumed to satisfy the following conditions:

(A1) $H_{1}(\gamma, p)$ and $H_{2}(\gamma, p)$ are continuous;

(A2) $H_{1}(\gamma, p)$ and $H_{2}(\gamma, p)$ are nondecreasing in $\gamma \in \mathbb{R}, \forall p \in \mathbb{R}^{n}$;

(A3) $H_{1}(\gamma, p)$ is convex and $H_{2}(\gamma, p)$ is concave in $p \in \mathbb{R}^{n}, \forall \gamma \in \mathbb{R}$;

(A4) $H_{1}(\gamma, p)$ and $H_{2}(\gamma, p)$ are positively homogeneous of degree 1 in $p \in \mathbb{R}^{n}$, i.e., $H_{1}(\gamma, \lambda p)=$ $\lambda H_{1}(\gamma, p), \forall \lambda \geq 0$. 
The LSC viscosity solution of (65) is given by

$$
u(x, y)=\inf _{y \in \mathbb{R}^{n}}\left[f(y) \vee H_{1}^{\sharp}\left(\frac{x-y}{t}\right)\right],
$$

and the USC viscosity solution of (66) is

$$
u(x, y)=\sup _{y \in \mathbb{R}^{n}}\left[f(y) \wedge H_{2 \sharp}\left(\frac{x-y}{t}\right)\right],
$$

where the conjugate operators $H^{\sharp}$ and $H_{\sharp}$ are defined as

$$
\begin{aligned}
& H^{\sharp}(q)=\inf \left\{\gamma \in \mathbb{R}: H(\gamma, p) \geq\langle p, q\rangle, \forall p \in \mathbb{R}^{n}\right\}, \\
& H_{\sharp}(q)=\sup \left\{\gamma \in \mathbb{R}: H(\gamma, p) \leq\langle p, q\rangle, \forall p \in \mathbb{R}^{n}\right\} .
\end{aligned}
$$

Proof. The proof for Cauchy problem (65) corresponds to the one of Theorem 5.5 in [2]. Problem (66) is symmetrically obtained by considering the relationship [65]:

$$
[-H(-\gamma,-p)]^{\sharp}(z)=-\left[H_{\sharp}(\gamma, p)\right](z) .
$$

The simplest case of admissible (A1)-(A4) convex Hamiltonian corresponds to $H(\gamma, p)=$ $\gamma\|p\|$ such that, using Cauchy-Schwartz inequality, one gets

$$
H^{\sharp}(q)=\inf \{\gamma \in \mathbb{R}: \gamma\|p\| \geq\langle p, q\rangle\}=\|q\| .
$$

The associated concave Hamiltonian is given by $H(\gamma, p)=-\gamma\|p\|$, whose conjugate is also $H_{\sharp}(q)=\|q\|$. Using this case as a starting point, a prototype of PDE in the framework of operators in (max, min)-algebra can be defined

Definition 12 Given any continous and bounded function $f: E \rightarrow[a, b] \subset \mathbb{R}$, the canonic (Hamilton-Jacobi) PDE in (max, min)-morphology is defined as

$$
\begin{cases}\frac{\partial u}{\partial t}= \pm u\|\nabla u\|, & x \in E, \quad t>0 \\ u(x, 0)=f(x), & x \in E\end{cases}
$$

and its (unique weak) solutions at scale $t$ are given by

$$
\begin{aligned}
& u(x, t)=\sup _{y \in E}\left\{f(y) \wedge \frac{\|x-y\|}{t}\right\} \quad(\text { for }+ \text { sign }), \\
& u(x, t)=\inf _{y \in E}\left\{f(y) \vee \frac{\|x-y\|}{t}\right\} \quad(\text { for }- \text { sign }) .
\end{aligned}
$$


Therefore the viscosity solutions of Cauchy problem (72) are a supmin convolution and an infmax convolution using the conic structuring function $c_{\lambda}(x)$ given by $(77)$, where the scale parameter is here the time; i.e., $\lambda=t$. More precisely, we note that these solutions

$$
\begin{aligned}
& u(x, t)=\left(f \nabla\left(-c_{t}\right)\right)(x) \quad(\text { for }+ \text { sign }), \\
& u(x, t)=\left(f \triangle c_{t}\right)(x) \quad(\text { for }- \text { sign }),
\end{aligned}
$$

are neither dual nor adjoint in the sense of Section 2. Consequently their composition does not lead to opening or closing since for instance:

$$
\left(\left(f \nabla\left(-c_{t}\right)\right) \triangle c_{t}\right) \neq\left(\left(f \nabla\left(-c_{t}\right)\right) \triangle^{*}\left(-c_{t}\right)\right)
$$

The model (72) can be generalized to

$$
\frac{\partial u}{\partial t}= \pm \alpha u\|\nabla u\|, \quad x \in E, t>0
$$

with initial condition $u(x, 0)=f(x)$ and $\alpha>0$, such that we easily see that the corresponding solutions are

$$
\begin{aligned}
& u(x, t)=\left(f \nabla\left(-c_{\alpha t}\right)\right)(x) \quad(\text { for }+\operatorname{sign}), \\
& u(x, t)=\left(f \triangle c_{\alpha t}\right)(x) \quad(\text { for }-\operatorname{sign}),
\end{aligned}
$$

or in other words, multiplying $u$ by $\alpha$ involves a scaling in time by $\alpha$. This principle can be a clue to explore the notion of spatially adaptive ( $\max , \min$ )-operators based on using a scale depending on space $x$, i.e., a model of the form $u_{t}= \pm \alpha(x) u\|\nabla u\|$.

Let us mention an alternative natural generalization of the canonic PDE which involves raising function $u$ to power $P, P>0$, given by

$$
\frac{\partial u}{\partial t}= \pm u^{P}\|\nabla u\|, \quad x \in E, t>0
$$

again with initial condition $u(x, 0)=f(x)$. Now the corresponding viscosity solutions are

$$
\begin{array}{lll}
u(x, t) & =\sup _{y \in E}\left\{f(y) \wedge\left(-c_{P ; t}(x-y)\right)\right\} & (\text { for }+\operatorname{sign}), \\
u(x, t)=\inf _{y \in E}\left\{f(y) \vee\left(-c_{P ; t}(x-y)\right)\right\} & (\text { for }-\operatorname{sign}) .
\end{array}
$$

where

$$
c_{P ; t}(x)=-\frac{\|x\|^{\frac{1}{P}}}{t^{\frac{1}{P}}} .
$$

We note that $c_{P ; t}(x)$ is a concave function if $P \leq 1$ and quasiconcave if $P>1$ (see next Section). 
As a final variation of the model of the canonical PDE (72), we can consider the case where the Hamiltonians are replaced by either the pair

$$
\left.\begin{array}{l}
H_{1}(\gamma, p)=(\alpha+\gamma)\|p\| \\
H_{2}(\gamma, p)=-(\alpha+\gamma)\|p\|
\end{array}\right\} \Leftrightarrow H_{1}^{\sharp}(q)=H_{\sharp 2}(q)=\|q\|-\alpha ;
$$

or the pair

$$
\left.\begin{array}{l}
H_{1}(\gamma, p)=(\alpha-\gamma)\|p\| \\
H_{2}(\gamma, p)=-(\alpha-\gamma)\|p\|
\end{array}\right\} \Leftrightarrow H_{1}^{\sharp}(q)=H_{\sharp 2}(q)=\alpha-\|q\| ;
$$

which involves respectively the structuring functions:

$$
c_{\alpha, t}^{\vee}(x)=-\left(\frac{\|x\|}{t}-\alpha\right) \text { and } c_{\alpha, t}^{\wedge}(x)=-\left(\alpha-\frac{\|x\|}{t}\right) .
$$

These models are related to the notion of viscous morphology, discussed below.

Another interesting example is the following convex case: $H(\gamma, p)=e^{\gamma}\|p\|$ which satisfies all (A1)-(A4). One computes the conjugate $H^{\sharp}(q)$ as follows: the inequality $H(\gamma, p)=$ $e^{\gamma}\|p\| \geq\langle p, q\rangle, \forall p \in \mathbb{R}^{n}$, holds if and only if $e^{\gamma} \geq\|q\|$, or $\gamma \geq \log \|q\|$. Thus $H^{\sharp}(q)=\log \|q\|$. For the corresponding concave $H(\gamma, p)=-e^{-\gamma}\|p\|$, using relationship (71), we have that $H_{\sharp}(q)=-\log \|q\|$.

Finally, let us bring here the case of a convex-concave Hamiltonian which leads to a curious pair of composed operators. Consider a Cauchy problem of the form

$$
\begin{cases}u_{t}+H(u, D u), & \text { in }(x, t) \in \mathbb{R}^{n} \times(0, \infty), \\ u(x, 0)=u_{0}(x), & \forall x \in \mathbb{R}^{n},\end{cases}
$$

where the Hamiltonian is given by $H(\gamma, p)=H_{1}(\gamma, p)+H_{2}(\gamma, p)$, such that $H_{1}$ and $H_{2}$ hold assumptions (A1)-(A4). In particular $H_{1}(\gamma, \cdot)$ is convex and $H_{2}(\gamma, \cdot)$ is concave. As shown in [65], the expected subsolution and supersolution are given by

$$
\begin{aligned}
& u_{-}(x, t)=\sup _{z \in \mathbb{R}^{n}} \inf _{y \in \mathbb{R}^{n}}\left\{u_{0}(x-t(y+z)) \vee H_{1}^{\sharp}(y) \wedge H_{2 \sharp}(z)\right\}, \\
& u_{+}(x, t)=\inf _{z \in \mathbb{R}^{n}} \sup _{y \in \mathbb{R}^{n}}\left\{u_{0}(x-t(y+z)) \wedge H_{2 \sharp}(y) \vee H_{1}^{\sharp}(z)\right\} .
\end{aligned}
$$

In addition, if $u_{0}$ is a bounded and uniformly continuous function, this problem admits a unique viscosity solution $u(x, t)$ such that $u_{-} \leq u \leq u_{+}$.

As example of application, we can consider the following Cauchy problem introduced in [65]:

$$
\begin{cases}\frac{\partial u}{\partial t}=-\|\nabla u\| \sinh u, & x \in E, t>0 \\ u(x, 0)=f(x), & x \in E\end{cases}
$$

where $\sinh a$ is the hyperbolic $\sin$ function; i.e., $\sinh a=1 / 2\left(e^{a}-e^{-a}\right), \forall a \in \mathbb{R}$. The Hamiltonian $H(\gamma, p)=\|p\| \sinh \gamma$ can be written as $H(\gamma, p)=H_{1}(\gamma, p)+H_{2}(\gamma, p)=1 / 2 e^{\gamma}\|p\|$ 
$-1 / 2 e^{-\gamma}\|p\|$, meeting the assumption of sum of convex and concave functions. As we have shown above: $H_{1}^{\sharp}(q)=\log (2\|q\|)$ and $H_{2 \sharp}(q)=-\log (2\|q\|)$. Hence the subsolution and supersolution are derived from (80) and (81); i.e.,

$$
\begin{aligned}
& u_{-}(x, t)=\sup _{z \in \mathbb{R}^{n}} \inf _{y \in \mathbb{R}^{n}}\{f(x-t(y+x)) \vee \log (2\|y\|) \wedge-\log (2\|z\|)\}, \\
& u_{+}(x, t)=\inf _{z \in \mathbb{R}^{n}} \sup _{y \in \mathbb{R}^{n}}\{f(x-t(y+x)) \wedge-\log (2\|y\|) \vee \log (2\|z\|)\} .
\end{aligned}
$$

Using our notation, we have

$$
\begin{aligned}
& u_{-}(x, t)=\left(\left(f \triangle l_{t}\right) \nabla l_{t}\right)(x), \\
& u_{+}(x, t)=\left(\left(f \nabla l_{t}\right) \triangle l_{t}\right)(x),
\end{aligned}
$$

with $l_{t}(x)=-\log \left(2\|x\| t^{-1}\right)$. We can expect that, even if the solution of (82) is neither an opening nor a closing, it will produce a rather symmetric regularization of a bounded real valued function $f$. That corresponds to a kind of self-dual filtering with respect to 0 .

\section{Nonlinear analysis using operators $(\triangle, \nabla)$}

Besides their relationships with first-order Hamilton-Jacobi PDE, (max, min)-convolutions are powerful tools for other areas of nonlinear mathematics.

\subsection{Quasi-concavity}

Minkowski addition and subtraction behave particularly well for convex sets [46]. If $X$ is convex, $X \ominus B$ is convex for any $B$. If $X$ and $B$ are convex, $X \oplus B$ is convex. The interest of ( $\max ,+)$-convolutions (i.e., classical dilation $(f \oplus b)$ and erosion $(f \ominus b)$ of functions) in convex analysis is known from the origin of this area of mathematics [49, 55]. Convolutions in (max, min)-algebra play a similar, but less known, role in the case of a more general class of functions: the quasiconvex functions.

Let us start by a recall on quasiconvexity/quasiconcavity [9]. A function $f \in \mathcal{F}(C, \overline{\mathbb{R}})$ defined on a convex set $C \subset \mathbb{R}^{n}, C \in \mathcal{C}$, is said to be quasiconvex if, for every $x, y \in \mathbb{R}^{n}$ and $\lambda \in(0,1)$, one has

$$
f(\lambda x+(1-\lambda) y) \leq f(x) \vee f(y) .
$$

On the contrary, $f \in \mathcal{F}(C, \overline{\mathbb{R}})$ is said to be quasiconcave if

$$
f(\lambda x+(1-\lambda) y) \geq f(x) \wedge f(y) .
$$

Note that $f$ is quasiconvex if and only if $-f$ (or $M-f$ in the case of functions bounded in $[0,1])$ is quasiconcave. Roughly speaking, we can said that any "unimodal function" is quasiconcave. 
An alternative way of defining quasiconvexity and quasiconvexity involves level sets. A function $f \in \mathcal{F}(C, \overline{\mathbb{R}})$ is quasiconvex if every lower level set of $f$ is convex, i.e., $X_{h}^{-}(f) \in \mathcal{C}$. A function $f \in \mathcal{F}(C, \overline{\mathbb{R}})$ is quasiconcave if every upper level set of $f$ is convex $X_{h}^{+}(f) \in \mathcal{C}$. Using formulas (14) and (15), we note that the definition of quasiconvexity is valid by replacing the convexity of lower level set by the convexity of strict lower level set.

The notion of quasiconcavity is weaker than the notion of concavity, in the sense that every concave function is quasiconcave. Similarly, every convex function is quasiconvex.

Note also that a concave function can be a quasiconvex function, e.g., $x \mapsto \log (x)$ is concave, and it is quasiconvex.

We can now introduce the following immediate result.

Proposition 13 Let $b: C \rightarrow \overline{\mathbb{R}}$ be a quasiconcave function defined on a convex set $C \subset \mathbb{R}^{n}$, $C \in \mathcal{C}$.

- If $g \in \mathcal{F}(C, \overline{\mathbb{R}})$ is a quasiconvex function, then the infmax convolution $(g \triangle b)$ is quasiconvex.

- If $f \in \mathcal{F}(C, \overline{\mathbb{R}})$ is a quasiconcave function, then the supmin convolution $(f \nabla b)$ is quasiconcave.

Proof. The proof is straightforward from the formulation of supmin and infsup convolutions in terms of respectively Minkowski sum of upper level sets and lower level sets; i.e., expressions (30) and (31), together with the property of convexity preservation of Minkowski addition $X \oplus B$ if both $X$ and $B$ are convex.

In addition, if we observe the expressions (32) and (33) of the adjoint operators, which involve Minkowski substraction, we have a straightforward more general result:

- If $g \in \mathcal{F}(C, \overline{\mathbb{R}})$ is a quasiconvex function, then the adjoint infmax convolution $\left(g \triangle^{*} b\right)$ is quasiconvex for any structuring function $b \in \mathcal{F}(C, \overline{\mathbb{R}})$.

- If $f \in \mathcal{F}(C, \overline{\mathbb{R}})$ is a quasiconcave function, then the adjoint supmin convolution $\left(f \nabla^{*} b\right)$ is quasiconcave for any structuring function $b \in \mathcal{F}(C, \overline{\mathbb{R}})$.

As a consequence, for the product operators, openings and closings, we also have nice properties of quasiconcavity/convexity preservation.

Proposition 14 Let $b: C \rightarrow \overline{\mathbb{R}}$ be a quasiconcave function defined on a convex set $C \subset \mathbb{R}^{n}$, $C \in \mathcal{C}$.

- If $g \in \mathcal{F}(C, \overline{\mathbb{R}})$ is a quasiconcave function, then the (max, min)-opening $(g \triangleright b)$ is quasiconcave.

- If $f \in \mathcal{F}(C, \overline{\mathbb{R}})$ is a quasiconvex function, then the (max, $\min )$-closing $(f \bullet b)$ is quasiconvex. 
Proof. In both cases the result is trivial from the expressions of (max, min)-opening and closing in terms of level sets. For instance, one has for the closing:

$$
(f \diamond b)=\left(\left(f \nabla^{*} b\right) \triangle b\right),
$$

So, if $b$ is quasiconcave then $\check{b}^{c}$ is quasiconvex, then as $f$ is quasiconvex, the operator $\left(f \nabla^{*} b\right)$ is quasiconvex, so $\left(\left(f \nabla^{*} b\right) \triangle b\right)$ too.

The study of connectedness of level sets under ( $\max , \min )$-convolutions is also straightforward from the properties of connectedness of Minkowski addition; i.e., if $X$ and $B$ are connected, $X \oplus B$ is connected [46]. The following assertions hold [39]: (i) if $f$ and $b$ have connected strict lower (resp. strict upper) sets then so does $(f \triangle b)$ (resp. $(f \nabla b)$ ); (ii) if $f$ and $b$ have connected lower (resp. upper) sets then so does $(f \triangle b)(\operatorname{resp} .(f \nabla b))$. Other issues related to connectedness, as the existence of nonlocal minima of $(f \triangle b)(x)$ or nonlocal maxima of $(f \nabla b)(x)$ as well as some properties on continuity of level set maps from (max, min)-convolutions have been also studied in [39].

\subsection{Lipschitz approximation}

In many mathematical areas, from nonlinear analysis to optimization theory, it is important to construct Lipschitz approximation of a given function. We need first to recall the notion of Lipschitzian function. A function $\phi: \mathbb{R}^{n} \rightarrow \mathbb{R}$ is said to be Lipschitz with constant $L \in \mathbb{R}_{+}$ if

$$
|\phi(x)-\phi(y)| \leq L\|x-y\|, \quad \forall x, y \in \mathbb{R}^{n} .
$$

In this respect, (max, min)-convolutions has a fruitful role to play [57].

Proposition 15 Let the structuring function $b: \mathbb{R}^{n} \rightarrow \mathbb{R}$ be majorized from above and $L$ Lipschitz.

- If $f: \mathbb{R}^{n} \rightarrow \mathbb{R} \cup\{-\infty\}$ is a proper function, then the supmin convolution $(f \nabla b)$ is L-Lipschitz.

- If $g: \mathbb{R}^{n} \rightarrow \mathbb{R} \cup\{+\infty\}$ is a proper function, then the infmax convolution $(g \triangle b)$ is L-Lipschitz.

Proof. We provide the proof for the supmin convolution; the case of infmax is obtained similarly. First, the operator can be written in the form

$$
(f \nabla b)(x)=\sup \left\{\phi_{y}(x): y \in \operatorname{dom}^{-} f\right\},
$$

where

$$
\phi_{y}(x)=f(y) \vee b(x-y) .
$$


Obviously, since $b(x)$ is $L$-Lipschitz, for each $y \in \operatorname{dom}^{-} f$, the function $\phi_{y}: \mathbb{R}^{n} \rightarrow \mathbb{R}$ is also $L$-Lipschitz. Indeed, for all $x$ and $z$ in $\mathbb{R}^{n}$, we can write

$$
\begin{aligned}
\left|\phi_{y}(x)-\phi_{y}(z)\right| & =|f(y) \vee b(x-y)-f(y) \vee b(z-y)| \\
& \leq|b(x-y)-b(z-y)| \\
& \leq L\|x-z\| .
\end{aligned}
$$

This is equivalent to say that

$$
\phi_{y}(z)-L\|x-z\| \leq \phi_{y}(x) \leq \phi_{y}(z)+L\|x-z\|,
$$

and then, taking the supremum with respect to $y \in \operatorname{dom}^{-} f$, one gets

$$
(f \nabla b)(z)-L\|x-z\| \leq(f \nabla b)(x) \leq(f \nabla b)(z)+L\|x-z\|
$$

which is equivalent

$$
|(f \nabla b)(x)-(f \nabla b)(z)| \leq L\|x-z\|
$$

Roughly speaking, this basic result asserts that the supmin and infmax convolutions of $f$ and $g$ by $b$ inherits the Lipschitzian property of $b$, no matter how bad the functions $f$ and $g$ are.

A well-known case of Lipschitzian function is the conic structuring function:

$$
c_{\lambda}(x)=-\lambda^{-1}\|x\|, \quad \lambda>0,
$$

which is just a $\lambda^{-1}$-Lipschitz function. By using our canonic multiscale structuring function $c_{\lambda}$ relevant properties of regularization are obtained.

Proposition 16 Let $f$ be a nonpositive proper function and $g$ be a nonnegative proper function.

- (Lipschitzian approximation) The supmin convolution $f^{[\lambda]}(x)=\left(f \nabla c_{\lambda}\right)(x)$ and the infmax convolution $g_{[\lambda]}(x)=\left(g \triangle c_{\lambda}\right)(x)$ are $\lambda^{-1}$-Lipschitz.

- (Convergence to envelopes) The lower envelope of the family $\left\{f^{[\lambda]}: \lambda>0\right\}$ converges monotonically downwards to the upper-semicontinuous hull $\mathrm{cl}^{+} f$ of $f$, i.e.,

$$
\left(\mathrm{cl}^{+} f\right)(x)=\inf _{\lambda>0} f^{[\lambda]}(x), \forall x \in \mathbb{R}^{n} .
$$

The upper envelope of the family $\left\{g_{[\lambda]}: \lambda>0\right\}$ converges monotonically upwards to the lower-semicontinuous hull $\mathrm{cl}^{-} g$ of $g$, i.e.,

$$
\left(\mathrm{cl}^{-} g\right)(x)=\sup _{\lambda>0} g^{[\lambda]}(x), \forall x \in \mathbb{R}^{n} .
$$


- (Local extrema preservation) For $\left.f\left(x_{0}\right) \in\right]-\infty, 0[$, one has the equivalence

$$
f^{[\lambda]}\left(x_{0}\right)=f\left(x_{0}\right) \quad \Longleftrightarrow \quad x_{0} \text { maximizes } f \text { over } \stackrel{\circ}{B}_{\lambda f\left(x_{0}\right)}\left(x_{0}\right)
$$

For $\left.g\left(x_{0}\right) \in\right] 0,+\infty,[$, one has the equivalence

$$
g_{[\lambda]}\left(x_{0}\right)=g\left(x_{0}\right) \quad \Longleftrightarrow \quad x_{0} \text { minimizes } g \text { over } \stackrel{\circ}{B}_{\lambda g\left(x_{0}\right)}\left(x_{0}\right)
$$

where $\stackrel{\circ}{B}_{r}(x)$ is the open ball centered at $x$ and radius $r>0$.

Proof. Lipschitzian approximation is a particular case of previous proposition. The proof of convergence of infmax convolution corresponds to that of Proposition 3.2 in [57]. For the supmin convolution the same result is obtained by duality.

Let us show how local minima preservation holds, which is based on the proof of Proposition 3.3 in [57]. On the one hand, recall that one has always the inequality $g_{[\lambda]}\left(x_{0}\right) \leq g\left(x_{0}\right)$. On the other hand,

$$
g\left(x_{0}\right) \leq g(y) \vee \lambda^{-1}\left\|x_{0}-y\right\|, \quad \forall y \in \mathbb{R}^{n},
$$

which is equivalent to

$$
g\left(x_{0}\right) \leq g(y) \text { whenever } \lambda^{-1}\left\|x_{0}-y\right\|<g\left(x_{0}\right) .
$$

Therefore, $g_{[\lambda]}\left(x_{0}\right)=g\left(x_{0}\right)$ if and only if $g(y) \geq g\left(x_{0}\right)$ for all $y \in \stackrel{\circ}{B}_{\lambda g\left(x_{0}\right)}\left(x_{0}\right)$.

The local maxima preservation is obtained analogously, since we have

$$
f\left(x_{0}\right) \geq f(y) \wedge \lambda^{-1}\left\|x_{0}-y\right\|, \quad \forall y \in \mathbb{R}^{n}, \quad \Rightarrow \quad f\left(x_{0}\right) \geq f(y) \text { whenever } \lambda^{-1}\left\|x_{0}-y\right\|<f\left(x_{0}\right)
$$

which together with inequality $f^{[\lambda]}\left(x_{0}\right) \geq f\left(x_{0}\right)$, involves that $f^{[\lambda]}\left(x_{0}\right)=f\left(x_{0}\right)$ if and only if $f(y) \leq f\left(x_{0}\right)$ for all $y \in \stackrel{\circ}{B}_{\lambda f\left(x_{0}\right)}\left(x_{0}\right)$.

Regularization effects of supmin and infmax convolutions by the conic structuring functions can be generalized to more general one-parameter kernels. For instance, for $\left(g \triangle k_{\lambda}\right)$, a kernel $k_{\lambda}: \mathbb{R}_{+} \rightarrow \overline{\mathbb{R}}$ of type $k_{\lambda}\left(\lambda^{-1}\|x\|\right)$ such that $k(\cdot)$ is nondecreasing, $k(0) \leq \max g$ and $\min g<\max k$; e.g., $k_{\lambda}(x)=\log \left(\lambda^{-1}\|x\|\right)$. See main generalized results in [53].

Bibliographic remark. The use of standard dilation and erosion for convex functions, i.e., $(\max ,+)$-convolutions, for regularization is well known. In particular, the MoreauYosida transform of a convex function $g$, which consists in an erosion (infimal convolution) of $g$ with a parabolic structuring function, produces a Lipschitz continuously differentiable approximation of the function. Note that for the case of a quasiconvex function it works only in the 1-dimensional case.

If we replace the convexity of $g$ by either a condition of boundness on bounded sets or a quadratic minorization property, one can obtain approximation and regularization of these 
arbitrary functions $g$ in Hilbert spaces by the Lasry-Lions method [36, 8], which consists in a quadratic erosion followed by a quadratic dilation. This technique can be also applied to functions on a Riemannian manifold of nonpositive curvature [6].

The generalization of the (max, min)-regularization considered here to a counterpart of the Lasry-Lions framework as well as the extension to curved spaces will be the object of ongoing research.

\subsection{A transform in (max, $\min )$-convolution}

The Fourier transform of the classical convolution in $(+, \times)$ algebra of two functions is the product of their corresponding transforms. Similarly, in the context of convex analysis, the Legendre-Fenchel transform of the $(\max ,+)$ inf-convolution coincides with the sum of the corresponding Legendre-Fenchel transforms.

We recall the Legendre-Fenchel transform of a function $\phi: E \rightarrow \mathbb{R} \cup\{+\infty\}$ is the function $\phi^{*}: E^{*} \rightarrow \mathbb{R} \cup\{-\infty\}$ given by

$$
\phi^{*}(w)=\sup _{x \in E}\{\langle w, x\rangle-\phi(x)\}, \quad \forall w \in E^{*},
$$

where $E$ and its Fenchel conjugate space $E^{*}$ are subsets of $\mathbb{R}^{n}$. In that what follows, the symbol $\Lambda$ stands for the $2 \mathrm{D}$ simplex

$$
\Lambda=\left\{\left(\lambda_{1}, \lambda_{2}\right) \in \mathbb{R}^{2}: \lambda_{1}+\lambda_{2}=1, \lambda_{1} \geq 0, \lambda_{2} \geq 0\right\} .
$$

We need to introduce also the notion of right multiplication of $\phi^{*}$ by a finite scalar $\lambda \geq 0$ as follows

$$
\left(\phi^{*} \lambda\right)(w)= \begin{cases}\lambda \phi^{*}\left(\lambda^{-1} w\right) & \text { if } \lambda>0 \\ \sup _{x \in \operatorname{dom}^{-}{ }}\langle w, x\rangle & \text { if } \lambda=0\end{cases}
$$

Using these ingredients, relationship between Legendre-Fenchel transform and (max, min)convolutions was considered in [57]. We consider in particular the case for the infmax convolution. Let $f: E \rightarrow \mathbb{R} \cup\{+\infty\}$ be a proper convex function and $b: E \rightarrow \mathbb{R} \cup\{-\infty\}$ be a proper concave function. The Legendre-Fenchel transform of $(f \triangle b)$ is given by

$$
(f \triangle b)^{*}(w)=\inf _{\left(\lambda_{1}, \lambda_{2}\right) \in \Lambda}\left(f^{*} \lambda_{1}+\left(\check{b}^{c}\right)^{*} \lambda_{2}\right)(w), \quad \forall w \in E^{*} .
$$

Even if the formula (88) seems compact, it presents from our viewpoint a limited interest since, on the one hand, it is related to a representation of the infmax convolution as

$$
(f \triangle b)(x)=\inf _{(u, v) \in M} \sup _{\left(\lambda_{1}, \lambda_{2}\right) \in \Lambda}\left\{\lambda_{1} f(u)+\lambda_{2} \check{b}^{c}(v)\right\}
$$

where

$$
M=\left\{(u, v) \in \operatorname{dom}^{-} f \times \operatorname{dom}^{-} \check{b}^{c}: u+v=x\right\} .
$$


On the other hand, it is limited to convex functions.

Research on another transform intrinsically adapted to (max, $\min$ )-convolutions have been conducted in two independent approaches. Gondran in [26] introduced the so-called infmaxaffine transform; Volle in [68], inspired from the results on Hamilton-Jacobi PDE developed by Barron and co-workers [11, 12] (see previous Section), introduced quasiconvex conjugate transform. We adopt here the framework developed by Volle and consequently proof of all results are given in [68]. We have nevertheless adapted the notation and terminology to our context.

Support function. The notion of support function plays a central role in the transform domain considered here. We recall that the support function of a nonempty set $X \subset \mathbb{R}^{n}$ is defined by

$$
i_{X}^{*}(y)=\sup _{x \in X}\langle x, y\rangle, \quad \forall y \in \mathbb{R}^{n},
$$

with the convention $\sup \emptyset=-\infty$. We note that the support function of $X$ is just the Legendre-Fenchel transform of the indicator function $i_{X}$ of $X$ :

$$
i_{X}(x)=\left\{\begin{array}{cl}
0 & \text { if } x \in X \\
+\infty & \text { if } x \in X^{c}
\end{array}\right.
$$

As examples, we can mention: i) the support function of a singleton $X=\{x\}$ is $i_{X}^{*}(\lambda y)=$ $\langle x, y\rangle$; ii) the support function of the Euclidean unit ball $X=B_{1}$ is $i_{X}^{*}(\lambda y)=\|x\|$.

The support function is positively homogenous, i.e., $i_{X}^{*}(\lambda y)=\lambda i_{X}^{*}(y) \lambda>0, \forall y \in \mathbb{R}^{n}$ and subadditive, i.e., $i_{X}^{*}(y+z) \leq i_{X}^{*}(y)+i_{X}^{*}(z), \forall y, z \in \mathbb{R}^{n}$. It follows that $i_{X}^{*}$ is a LSC convex function.

The support functions of a scaled or translated set are closely related to the original set $X:$ i) $i_{\lambda X}(x)=\lambda i_{X}(x), \lambda>0, y \in \mathbb{R}^{n}$; ii) $i_{X+w}(x)=i_{X}(x)+\langle x, w\rangle, y, w \in \mathbb{R}^{n}$. The latter generalizes to one of the fundamental properties of the support function: the additivity with respect to the Minkowski addition, i.e., for all $y \in \mathbb{R}^{n}$ one has

$$
i_{X \oplus B}^{*}(y)=i_{X}^{*}(y)+. i_{B}^{*}(y),
$$

where $(+\infty)+.(-\infty)=(-\infty)+.(+\infty)=-\infty$.

$\alpha$ and $\beta$-conjugates. The motivation of the conjugate domain in ( $\max , \min )$ algebra is founded on the representation of infmax convolution as the Minkowski addition of lower level sets. In fact, the corresponding representation is just based on the support function of each lower level set.

Definition 17 To each extended real-valued function $f: \mathbb{R}^{n} \rightarrow \overline{\mathbb{R}}$, let us associate the $\alpha$ conjugate function $f^{\alpha}: \mathbb{R}^{n} \times \mathbb{R} \rightarrow \overline{\mathbb{R}}$ defined by

$$
f^{\alpha}(y, h)=\sup _{f(x)<h}\langle x, y\rangle=i_{Y_{h}^{-}(f)}^{*} .
$$


For any $y \in \mathbb{R}^{n}, f^{\alpha}(y, \cdot)$ is LSC and nondecreasing. Moreover, for any family $\left(f_{i}\right)_{i \in I}, f_{i} \in$ $\mathcal{F}\left(\mathbb{R}^{n}, \overline{\mathbb{R}}\right)$, one has

$$
\left(\inf _{i \in I} f_{i}\right)^{\alpha}=\sup _{i \in I} f_{i}^{\alpha} .
$$

This $\alpha$-conjugation is closely related to the infsup convolution [68](Theorem 3.1):

Proposition 18 Given any pair of functions $f, b \in \mathcal{F}\left(\mathbb{R}^{n}, \overline{\mathbb{R}}\right)$, one always has

$$
\left(f \triangle \check{b}^{c}\right)^{\alpha}=f^{\alpha}+. b^{\alpha} .
$$

This result is an easy consequence of relation (31) and the property (90):

$$
\begin{aligned}
(f \triangle b)^{\alpha}(y, h) & =i_{Y_{h}^{-}(f \triangle b)}^{*}(y)=i_{Y_{h}^{-}(f) \oplus Y_{h}^{-}\left(\check{b}^{c}\right)}^{*}=i_{Y_{h}^{-}(f)}^{*}(y)+. i_{Y_{h}^{-}\left(\check{b}^{c}\right)}^{*}(y) \\
& =f^{\alpha}(y, h)+.\left(\check{b}^{c}\right)^{\alpha}(y, h) .
\end{aligned}
$$

Let us define now a kind of dual (pseudo-inverse) transform.

Definition 19 The $\beta$-conjugate of any function $\phi: \mathbb{R}^{n} \times \mathbb{R} \rightarrow \overline{\mathbb{R}}$ is the extended real-valued function $\phi^{\beta}: \mathbb{R}^{n} \rightarrow \overline{\mathbb{R}}$ defined as

$$
\phi^{\beta}(x)=\sup _{y \in \mathbb{R}^{n}} \sup \{h \in \mathbb{R}:\langle x, y\rangle>\phi(y, h)\} .
$$

We also have the property

$$
\left(\inf _{i \in I} \phi_{i}\right)^{\beta}=\sup _{i \in I} \phi_{i}^{\beta}
$$

for any family $\left(\phi_{i}\right)_{i \in I}, \phi_{i} \in \mathcal{F}\left(\mathbb{R}^{n} \times \mathbb{R}, \overline{\mathbb{R}}\right)$.

The $\alpha$ and $\beta$ conjugates are related by the following Galois correspondence

$$
f^{\alpha} \leq \phi \Leftrightarrow f \geq \phi^{\beta}
$$

for any pair of functions $f \in \mathcal{F}\left(\mathbb{R}^{n}, \overline{\mathbb{R}}\right)$ and $\phi \in \mathcal{F}\left(\mathbb{R}^{n} \times \mathbb{R}, \overline{\mathbb{R}}\right)$.

We consider the composite operators, called the biconjugates

$$
\begin{aligned}
f \in \mathcal{F}\left(\mathbb{R}^{n}, \overline{\mathbb{R}}\right) & \mapsto f^{\alpha \beta}=\left(f^{\alpha}\right)^{\beta} \in \mathcal{F}\left(\mathbb{R}^{n}, \overline{\mathbb{R}}\right), \\
\phi \in \mathcal{F}\left(\mathbb{R}^{n} \times \mathbb{R}, \overline{\mathbb{R}}\right) & \mapsto \phi^{\beta \alpha}=\left(\phi^{\beta}\right)^{\alpha} \in \mathcal{F}\left(\mathbb{R}^{n} \times \mathbb{R}, \overline{\mathbb{R}}\right) .
\end{aligned}
$$

The biconjugates are

- increasing: $f \leq g \Rightarrow f^{\alpha \beta} \leq g^{\alpha \beta} ; \phi \leq \psi \Rightarrow \phi^{\beta \alpha} \leq \psi^{\beta \alpha}$;

- antiextensive: $f^{\alpha \beta} \leq f ; \phi^{\beta \alpha} \leq \phi$;

- idempotent: $\left(f^{\alpha \beta}\right)^{\alpha \beta}=f^{\alpha \beta} ;\left(\phi^{\beta \alpha}\right)^{\beta \alpha}=\phi^{\beta \alpha}$. 
The biconjugate of $f \in \mathcal{F}\left(\mathbb{R}^{n}, \overline{\mathbb{R}}\right)$, resp. $\phi$, is nothing but the greatest regular minorant of $f$, resp. $\phi \in \mathcal{F}\left(\mathbb{R}^{n} \times \mathbb{R}, \overline{\mathbb{R}}\right)$ :

$$
\begin{aligned}
& f^{\alpha \beta}=\sup \left\{g \in \mathcal{F}\left(\mathbb{R}^{n}, \overline{\mathbb{R}}\right): g^{\alpha \beta}=g \text { and } g \leq f\right\}, \\
& \phi^{\beta \alpha}=\sup \left\{\psi \in \mathcal{F}\left(\mathbb{R}^{n}, \overline{\mathbb{R}}\right): \psi^{\beta \alpha}=\psi \text { and } \psi \leq \phi\right\} .
\end{aligned}
$$

All the above properties are mere consequences of the theory of Galois correspondences.

We can introduce now one of the important results which describes the classes of regular functions in such conjugate domains [68](Theorem 3.4).

Proposition 20 Let $f: \mathbb{R}^{n} \rightarrow \overline{\mathbb{R}}$ be an extended real-valued function. Then

$$
f=f^{\alpha \beta}
$$

if and only if $f$ is a LSC quasiconvex function.

From the previous results, we observe that for any pair of functions $f, b \in \mathcal{F}\left(\mathbb{R}^{n}, \overline{\mathbb{R}}\right)$, $\left(f^{\alpha}+. b^{\alpha}\right)^{\beta}$ is just the LSC quasiconvex hull of the infmax convolution $f \triangle \check{b}^{c}$. In the case when $f$ and $b$ are quasiconvex, $f \triangle \check{b}^{c}$ is quasiconvex too so that $\left(f^{\alpha}+. b^{\alpha}\right)^{\beta}$ is then the LSC hull of $f \triangle \check{b}^{c}$. In order to have the explicit equivalence $f \triangle \check{b}^{c}=\left(f^{\alpha}+. b^{\alpha}\right)^{\beta}, f$ and $b$ should be quasiconvex, compact and satisfy some other minor technical constraints, see [68](Theorem $4.2)$.

Applications. Let us come back to the first-order Hamilton-Jacobi PDE discussed in Proposition 11. We remind that the LSC viscosity solution with convex Hamiltonian $H(\gamma, p)$, $p \in \mathbb{R}^{n}, \gamma \in \mathbb{R}$, involves the conjugate Hamiltonian (69), given by

$$
H^{\sharp}(q)=\inf \left\{\gamma \in \mathbb{R}: H(\gamma, p) \geq\langle p, q\rangle, \forall p \in \mathbb{R}^{n}\right\} .
$$

As $H(\cdot, p)$ is nondecreasing, we note that the function $H^{\sharp}(q)$ above is just the $\beta$-conjugate of Hamiltonian $H(\gamma, p)$, namely:

$$
H^{\sharp}(q)=(H(p, \gamma))^{\beta}(q)=\sup _{p \in \mathbb{R}^{n}} \sup \{\gamma \in \mathbb{R}: H(p, \gamma)<\langle p, q\rangle\} .
$$

As we have discussed in Section 4, for the case $H(p, \gamma)=\gamma\|p\|$, we have $H^{\beta}(q)=\|p\|$. Therefore, $\alpha$ and $\beta$ conjugates can be applied to solve some Hamilton-Jacobi PDEs.

We conclude this part by describing the conjugates of an important class of quasiconvex functions, namely the radial quasiconvex functions, which generalizes the previous case. Let us consider that we work in a general Banach space $E$ equipped with a norm $\|\cdot\|$ and denote by $\|\cdot\|_{*}$ the dual norm:

$$
\|\cdot\|_{*}(y)=\max _{\|x\|=1}\langle x, y\rangle
$$


for any $y$ in the topological dual $F$ of $E$. We remind that if $p, q \in[1,+\infty]$ satisfy $1 / p+1 / q=1$, then the $L^{p}$ and $L^{q}$ norms are dual to each other. In particular, the Euclidean norm is selfdual $(p=q=2)$. To any nondecreasing extended real-valued function $a:[0,+\infty[\rightarrow \overline{\mathbb{R}}$ is associated the quasiconvex function $f=a \circ\|\cdot\|$, where $\circ$ denotes the composition of functions. Setting

$$
\tilde{a}(r)= \begin{cases}a(0) & \text { for } r \in]-\infty, 0[ \\ a(r) & \text { for } r \in[0,+\infty[\end{cases}
$$

we get a nondecreasing function $\tilde{a}: \mathbb{R} \rightarrow \overline{\mathbb{R}}$ such that $\tilde{a}(0)=\inf \tilde{a}$. We will use also the notion of LSC quasi-inverse of $a$ given by

$$
a^{e}(s)=\sup \{r \in \mathbb{R}: a(r)<s\}=\inf \{r \in \mathbb{R}: a(r) \geq s\} .
$$

The $\alpha$-conjugate of a radial quasiconvex function can be written as the product of a nondecreasing function by the dual norm. More precisely, we have the following result [68](Propositions 4.10 and 4.11):

Proposition 21 Let a and $\tilde{a}$ as above. Then, one has

$$
(a \circ\|\cdot\|)^{\alpha}(y, h)=\tilde{a}^{e}(h)\|y\|_{*}, \forall y \in F, h \in \mathbb{R} .
$$

Let $\phi: F \times \mathbb{R} \rightarrow \overline{\mathbb{R}}$ by defined by $\phi(y, h)=b(h)\|y\|_{*}$ with $b: \mathbb{R} \rightarrow \overline{\mathbb{R}}$ nondecreasing, then

$$
\phi^{\beta}(x)=b^{e}(\|x\|), \forall x \in E .
$$

Finally, we have the following result on biconjugates [68](Corollaries 4.12 and 4.13):

Proposition 22 Let $f=a \circ\|\cdot\|$ with function $a:\left[0,+\infty\left[\rightarrow \overline{\mathbb{R}}\right.\right.$ non decreasing. Then $f^{\alpha \beta}=$ $\bar{a} \circ\|\cdot\|$, where $\bar{a}$ is the LSC hull of a. Let $\phi: F \times \mathbb{R} \rightarrow \overline{\mathbb{R}}, \phi(y, h)=b(h)\|y\|_{*}$ with $b: \mathbb{R} \rightarrow \overline{\mathbb{R}}$ nondecreasing, the $\phi^{\beta \alpha}=\bar{b}(h)\|y\|_{*}$.

Let us conclude by saying that the theory of $\alpha, \beta$-conjugates and in particular, the considered case of radial quasiconvex functions, will be applied in the future to identify the interesting cases of structuring functions and their composition rules in the framework of (max, min)-convolutions.

Bibliographic remark. In the state-of-the-art of mathematical morphology, the notion of Legendre-Fenchel transform was rediscovered and generalized to any function, either convex or non convex: it is named the slope transform [22, 41].

Support function defined on convex sets in the plane was used by Schmitt [61] to formulate Minkowski addition of sets represented by its boundary. 


\section{Ubiquity of ( $\max , \min )$-convolutions in mathematical mor- phology}

Our aim in this Section is to review some previous approaches of "unconventional" dilation and erosion (in the sense that they cannot be related to $(\max ,+)$-convolutions paradigm) which fit naturally in a formulation as (max, min)-convolutions.

The two first cases are conventional and rather trivial, however, we consider that links to the other frameworks deserves some discussion.

\subsection{Distance function}

The distance function is a powerful tool in image processing. It is based on endowing space $E$ with a particular norm $\|\cdot\|$. Then for any nonempty subset $X \subset E$, the distance function of set $X, d_{X}: E \rightarrow \mathbb{R}_{+}$, is defined as

$$
d_{X}(x)=\inf _{y \in X^{c}}\|x-y\|, \quad x \in E .
$$

The distance function is just a particular case of the infmax convolution. Indeed, if one introduces the zero indicator function $\mathbb{D}_{X}$ of set $X$ :

$$
\mathbb{D}_{X}(x)=\left\{\begin{array}{cl}
0 & \text { if } x \in X \\
-\infty & \text { if } x \in X^{c}
\end{array}\right.
$$

then, using the unit conic structuring function $c_{1}(x)=-\|x\|$, one can write:

$$
d_{X}(x)=\left(-\mathbb{O}_{X^{c}} \triangle c_{1}\right)(x) .
$$

The proof is straightforward:

$$
\begin{aligned}
& \left(-\mathbb{O}_{X^{c}} \triangle c_{1}\right)(x)=\inf _{y \in E}\left\{-\mathbb{O}_{X^{c}}(y) \vee\|x-y\|\right\}=\left\{\begin{array}{cl}
\inf _{y \in E}\|x-y\| & \text { if } y \in X^{c} \\
+\infty & \text { if } y \in X
\end{array}\right. \\
& =\inf _{y \in X^{c}}\|x-y\| .
\end{aligned}
$$

By changing the norm in the conic unit structuring function, other distance functions can be obtained. Nevertheless, this infmax convolution is not the most efficient way to compute the distance function.

\subsection{Flat morphological operators using indicator functions}

The binary case of morphological operators is solved by simply using the standard indicator function $\mathbb{1}_{X}$ of a set $X \subset E$ :

$$
\mathbb{1}_{X}(x)= \begin{cases}1 & \text { if } x \in X \\ 0 & \text { if } x \in X^{c}\end{cases}
$$


Then, given a set $X$ and a structuring element $B$, we have

$$
\begin{aligned}
\left(\mathbb{1}_{X} \nabla \mathbb{1}_{B}\right) & =\mathbb{1}_{X \oplus B}, \\
\left(\mathbb{1}_{X} \triangle^{*} \mathbb{1}_{B}\right) & =\mathbb{1}_{X \ominus B} .
\end{aligned}
$$

This equivalence can be proven, without using upper level set representation, as follows:

$$
\begin{aligned}
\left(\mathbb{1}_{X} \nabla \mathbb{1}_{B}\right)(x)=1 & \Leftrightarrow \exists y \in E,\left(\mathbb{1}_{X}(y) \wedge \mathbb{1}_{B}(x-y)\right)=1, \\
& \Leftrightarrow \mathbb{1}_{X}(y)=1 \text { and } \mathbb{1}_{B}(x-y)=1, \\
& \Leftrightarrow y \in X \text { and } x-y \in B \\
& \Leftrightarrow x \in X \oplus B .
\end{aligned}
$$

Proof of the second equality can be carried out analogously.

More generally, the case of flat dilation and erosion for functions is also easily obtained by using the infinity indicator function $\infty_{X}$ of set $X$ :

$$
\infty_{X}(x)=\left\{\begin{array}{lll}
+\infty & \text { if } x \in X \\
-\infty & \text { if } x \in X^{c}
\end{array}\right.
$$

In the case of nonnegative bounded functions, $+\infty$ is replaced by $M$ and $-\infty$ by 0 . Then, for any function $f$ in $\mathcal{F}(E, \overline{\mathbb{R}})$ or in $\mathcal{F}(E,[0, M])$, we have:

$$
\begin{aligned}
\left(f \nabla \infty_{B}\right) & =(f \oplus B), \\
\left(f \triangle^{*} \infty_{B}\right) & =(f \ominus B) .
\end{aligned}
$$

The proof is trivial using the representation of the supmin and adjoint infmax convolutions in terms of upper level sets and taking into account that $X_{h}^{+}\left(\infty_{B}\right)=B, \forall h$; i.e.,

$$
\begin{aligned}
X_{h}^{+}\left(f \nabla_{B}\right) & =X_{h}^{+}(f) \oplus X_{h}^{+}\left(\infty_{B}\right)=X_{h}^{+}(f) \oplus B, \\
X_{h}^{+}\left(f \triangle^{*} \infty_{B}\right) & =X_{h}^{+}(f) \ominus X_{h}^{+}\left(\infty_{B}\right)=X_{h}^{+}(f) \ominus B .
\end{aligned}
$$

In addition, we also have that

$$
\begin{aligned}
\left(f \triangle \infty_{B}\right)= & \inf _{y \in E}\left\{f(y) \vee\left(-\check{\infty}_{B}\right)\right\}=(f \ominus B), \\
& \Rightarrow\left(f \triangle^{*} \infty_{B}\right)=\left(f \triangle \infty_{B}\right), \\
\left(f \nabla^{*} \infty_{B}\right)= & \sup _{y \in E}\left\{f(y) \vee^{*}\left(-\infty_{B}\right)\right\}=(f \oplus B), \\
& \Rightarrow\left(f \nabla^{*} \infty_{B}\right)=\left(f \nabla \infty_{B}\right) .
\end{aligned}
$$

and therefore for this case of flat structuring elements, which correspond to cylinders as equivalent structuring functions, the dual and adjoint operators play the same role. 


\subsection{Links with fuzzy morphology}

The state-of-the-art on morphological operators based on fuzzy logic is very extensive. We refer to papers by Nachtegael and Kerre [50] and by Bloch [14] which present an excellent survey of the existing literature on fuzzy morphology. The latter brightly discusses the role of duality with respect to complement vs. adjunction in fuzzy approaches. Results on fuzzy morphology discussed here are mainly based on Deng and Heijmans [20]. A complementary deeper insight from the algebraic viewpoint, in particular for representation theorems, can be found in a paper by Maragos [44].

In fuzzy logic, the two basic (Boolean) logic operators, the conjunction $C(s, t)=s \wedge t$ and the implication $I(s, t)=s \Rightarrow t(=\neg s \vee t)$, are extended from the Boolean domain $\{0,1\} \times\{0,1\}$ to the rectangle $[0,1] \times[0,1]$. A fuzzy conjunction is a mapping from $[0,1] \times[0,1]$ into $[0,1]$ which is increasing in both arguments and satisfies $C(0,0)=C(1,0)=C(0,1)=0$ and $C(1,1)=1$. A fuzzy implication is decreasing in the first argument, increasing in the second one and satisfies $I(0,0)=I(0,1)=I(1,1)=1$ and $I(1,0)=0$.

Given a fuzzy set $\mu$, the dilation and erosion by a fuzzy structuring element $\nu$ are then defined as [20]:

$$
\begin{aligned}
& \delta_{\nu, C}(\mu)(x)=\sup _{y}\{C(\nu(x-y), \mu(y))\}, \\
& \varepsilon_{\nu, C}(\mu)(x)=\inf _{y}\{I(\nu(y-x), \mu(y))\} .
\end{aligned}
$$

As shown in [20], $(I, C)$ is an adjunction if and only if $\left(\varepsilon_{\nu, C}, \delta_{\nu, C}\right)$ is an adjunction. Therefore, fuzzy opening and closing derived from the composition of these operators have all required properties, whatever the choice of the adjunction $(I, C)$. Note however that some properties such as the iteration; i.e.,

$$
\delta_{\nu_{2}, C}\left(\delta_{\nu_{1}, C}(\mu)\right)=\delta_{\delta_{\nu_{1}, C}\left(\nu_{2}\right), C}(\mu) ; \quad \varepsilon_{\nu_{2}, C}\left(\varepsilon_{\nu_{1}, C}(\mu)\right)=\varepsilon_{\delta_{\nu_{1}, C}\left(\nu_{2}\right), C}(\mu) ;
$$

require $C$ to be associative and commutative.

Two particular cases of conjunction and adjoint implication widely used in fuzzy logic are the Gödel-Brower:

$$
\begin{aligned}
C_{G B}(a, t) & =\min (a, t) \\
I_{G B}(a, t) & = \begin{cases}s, & s<a \\
1, & s \geq a\end{cases}
\end{aligned}
$$

and the Kleen-Dienes:

$$
\begin{aligned}
& C_{K D}(a, t)= \begin{cases}0, & t \leq 1-a \\
t, & t>1-a\end{cases} \\
& I_{K D}(a, s)=\max (1-a, s)
\end{aligned}
$$


It is consequently straightforward to see that the four operators that we have defined in Section 2 are just fuzzy dilations and erosions when they are applied to fuzzy sets (i.e., functions valued in $[0,1])$ :

$$
\begin{aligned}
& \delta_{\nu, C_{G B}}(\mu)(x)=(\mu \nabla \nu)(x) \stackrel{\text { adjoint }}{\longleftrightarrow} \varepsilon_{\nu, C_{G B}}(\mu)(x)=\left(\mu \triangle^{*} \nu\right)(x), \\
& \uparrow \text { dual } \uparrow \text { dual } \\
& \varepsilon_{\nu, C_{K D}}(\mu)(x)=(\mu \triangle \nu)(x) \stackrel{\text { adjoint }}{\longleftrightarrow} \delta_{\nu, C_{K D}}(\mu)(x)=\left(\mu \nabla^{*} \nu\right)(x) \text {. }
\end{aligned}
$$

Let us conclude clarifying that fuzzy logic is much more general than the pairs of conjunction/adjuntion considered here and consequently one cannot simply reduce fuzzy morphology to $(\max , \min )$-convolutions.

\subsection{Links with viscous morphology}

Theory and practice of morphological (flat) viscous operators was introduced by Vachier and Meyer [63, 64]. The PDE formulation of these operators was done by Maragos and Vachier [45]. The seminal idea was inspired from a physical model of image flooding by a viscous fluid [47].

As we have discussed at the beginning of the paper, a fundamental property of flat operators applied to numerical functions is the fact that they can be computed by applying the corresponding set operator to the upper level sets, followed by stacking the processed upper level sets. In other terms, the same set operator, that depends on a structuring element, acts equally for all upper level sets $X_{h}^{+}(f)$ : that can be interpreted as a similar behavior on all the structures whatever their intensity $h$. The idea of viscous operators is to apply a different scale (i.e., size) of structuring element at each upper level set. This principle can be seen now as an operator which locally adapts its activity with respect to the intensity.

Let us formalize their definition according to [45]. For the sake of simplicity, let us consider a nonnegative bounded function $f: E \rightarrow[0, M]$. Viscous operators have been formulated as isotropic transforms, that is based on the use of balls $B_{\lambda}$ as structuring elements. In this framework, the (isotropic) intensity-adaptive dilation and erosion of function $f$ are defined as

$$
\begin{aligned}
& \delta_{\lambda(h)}(f)=\sup \left\{h \in[0, M]: x \in\left(X_{h}^{+}(f) \oplus B_{\lambda(h)}\right)\right\}, \\
& \varepsilon_{\lambda(h)}(f)=\inf \left\{h \in[0, M]: x \in\left(X_{h}^{+}(f) \ominus B_{\lambda(h)}\right)\right\},
\end{aligned}
$$

where $\lambda(h):[0, M] \rightarrow[0, M]$ is the scaling function with respect to intensity $h$. Two types of viscosity (linear) functions have been proposed:

$$
\begin{aligned}
& \lambda_{\wedge}(h)=\lambda_{0} \frac{M-h}{M} \quad \text { (negative slope) } \\
& \lambda_{\vee}(h)=\lambda_{0} \frac{h}{M} \quad \text { (positive slope). }
\end{aligned}
$$


Note that, using $\lambda_{\wedge}(h)$, points of lowest intensity, $\lambda_{\wedge}(0)=\lambda_{0}$, are strongly dilated (resp. eroded) while points of highest intensity, $\lambda_{\wedge}(M)=0$, are left unchanged. Opposite effect are obtained for $\lambda_{\vee}(h)$. In the sequel, one set $\lambda_{0}=M$; i.e., $\lambda_{\wedge}(h)=M-h$ and $\lambda_{\vee}(h)=h$, such that $\lambda_{\wedge}$ and $\lambda_{\vee}$ are dual functions.

Using intensity-adaptive operators and the two viscosity functions, two pairs of viscous dilation and erosion are defined for a given function $f$ :

$$
\begin{aligned}
& \delta_{\wedge}^{\text {visc }}(f)=\delta_{\lambda_{\wedge}(h)}(f)=\sup \left\{h \in[0, M]: x \in\left(X_{h}^{+}(f) \oplus B_{M-h}\right)\right\}, \\
& \varepsilon_{\wedge}^{\text {visc }}(f)=\varepsilon_{\lambda_{\wedge}(h)}(f)=\sup \left\{h \in[0, M]: x \in\left(X_{h}^{+}(f) \ominus B_{M-h}\right)\right\},
\end{aligned}
$$

and

$$
\begin{aligned}
& \delta_{\vee}^{\mathrm{visc}}(f)=\delta_{\lambda_{\vee}(h)}(f)=\sup \left\{h \in[0, M]: x \in\left(X_{h}^{+}(f) \oplus B_{h}\right)\right\}, \\
& \varepsilon_{\vee}^{\text {visc }}(f)=\varepsilon_{\lambda_{\vee}(h)}(f)=\sup \left\{h \in[0, M]: x \in\left(X_{h}^{+}(f) \ominus B_{h}\right)\right\},
\end{aligned}
$$

such that $\left(\varepsilon_{\wedge}^{\text {visc }}, \delta_{\wedge}^{\text {visc }}\right)$ and $\left(\varepsilon_{\vee}^{\text {visc }}, \delta_{\vee}^{\text {visc }}\right)$ form two adjunctions. The pairs $\left(\varepsilon_{\vee}^{\text {visc }}, \delta_{\wedge}^{\text {visc }}\right)$ and $\left(\varepsilon_{\wedge}^{\text {visc }}, \delta_{\vee}^{\text {visc }}\right)$ are dual by complement. Consequently, their compositions produce two viscous openings and two closings: $\gamma_{\wedge}^{\text {visc }}(f)=\delta_{\wedge}^{\text {visc }}\left(\varepsilon_{\wedge}^{\text {visc }}(f)\right), \gamma_{\vee}^{\text {visc }}(f)=\delta_{\vee}^{\text {visc }}\left(\varepsilon_{\vee}^{\text {visc }}(f)\right), \varphi_{\wedge}^{\text {visc }}(f)=$ $\varepsilon_{\wedge}^{\text {visc }}\left(\delta_{\wedge}^{\text {visc }}(f)\right)$ and $\varphi_{\vee}^{\text {visc }}(f)=\varepsilon_{\vee}^{\text {visc }}\left(\delta_{\vee}^{\text {visc }}(f)\right)$.

If we introduce the following structuring function:

$$
v(x)= \begin{cases}M-\|x\| & \text { if }\|x\| \leq M \\ 0 & \text { if }\|x\|>M\end{cases}
$$

such that its complement structuring function is $v^{c}(x)=\|x\|$ if $\|x\| \leq M$ and $M$ if $\|x\|>M$, the previous viscous dilations and erosions (108)-(111) can be rewritten using the (max, min)convolution (respectively expressions (30), (32), (38), (39)):

$$
\begin{aligned}
& \delta_{\wedge}^{\text {visc }}(f)=\sup \left\{h \in[0, M]: x \in\left(X_{h}^{+}(f) \oplus X_{h}^{+}(v)\right)\right\}=(f \nabla v), \\
& \varepsilon_{\wedge}^{\text {visc }}(f)=\sup \left\{h \in[0, M]: x \in\left(X_{h}^{+}(f) \ominus X_{h}^{+}(v)\right)\right\}=\left(f \triangle^{*} v\right), \\
& \delta_{\vee}^{\text {visc }}(f)=\sup \left\{h \in[0, M]: x \in\left(X_{h}^{+}(f) \oplus Y_{h}^{-}\left(v^{c}\right)\right)\right\}=\left(f \nabla^{*} v\right), \\
& \varepsilon_{\vee}^{\text {visc }}(f)=\sup \left\{h \in[0, M]: x \in\left(X_{h}^{+}(f) \ominus Y_{h}^{-}\left(v^{c}\right)\right)\right\}=(f \triangle v) .
\end{aligned}
$$

The link between the corresponding viscous opening and closing and our (max, min)-opening and closing is trivial. In fact, using the results from Proposition 3, adjointness of two pairs of operators is obvious. Concerning the duality by complement, for instance, between $\delta_{\wedge}^{\text {visc }}(f)$ and $\varepsilon_{\vee}^{\mathrm{visc}}(f)$, we have, on the one hand,

$$
\begin{aligned}
\left(\delta_{\wedge}^{\mathrm{visc}}\left(f^{c}\right)\right)^{c} & =\left[\sup \left\{h \in[0, M]: x \in\left(X_{h}^{+}\left(f^{c}\right) \oplus X_{h}^{+}(v)\right)\right\}\right]^{c} \\
& =\inf \left\{(M-h) \in[0, M]: x \in\left(X_{h}^{+}\left(f^{c}\right) \oplus X_{h}^{+}(v)\right)\right\} \\
& =\inf \left\{(M-h) \in[0, M]: x \in\left(X_{h}^{+}(M-f) \oplus X_{h}^{+}(v)\right)\right\} \\
& =\inf \left\{h \in[0, M]: x \in\left(X_{M-h}^{+}(M-f) \oplus X_{M-h}^{+}(v)\right)\right\} \\
& =\inf \left\{h \in[0, M]: x \in\left(X_{h}^{-}(f) \oplus X_{h}^{-}\left(v^{c}\right)\right)\right\} .
\end{aligned}
$$


On the other hand, we have

$$
\begin{aligned}
\varepsilon_{\vee}^{\operatorname{visc}}(f) & =\sup \left\{h \in[0, M]: x \in\left(X_{h}^{+}(f) \ominus Y_{h}^{-}\left(v^{c}\right)\right)\right\} \\
& =\inf \left\{h \in[0, M]: x \notin\left(X_{h}^{-}(f) \ominus Y_{h}^{-}\left(v^{c}\right)\right)\right\} \\
& =\inf \left\{h \in[0, M]: x \in\left(Y_{h}^{-}(f) \oplus Y_{h}^{-}\left(v^{c}\right)\right)\right\} .
\end{aligned}
$$

Therefore, being precise, we have duality only when $\varepsilon_{\vee}^{\mathrm{visc}}(f)$ is exact, see (34). The difficulty to prove the duality by complement of viscous operators without the equivalence (36) was pointed out by Meyer [48].

In addition to the operator framework, a PDE formulation of viscous dilation and erosion was introduced in [45]. The two corresponding continuous models are:

$$
\begin{cases}\frac{\partial u}{\partial t}= \pm(\max f-u)\|\nabla u\|, & x \in E, t>0 \\ u(x, 0)=f(x), & x \in E\end{cases}
$$

and

$$
\begin{cases}\frac{\partial u}{\partial t}= \pm(u-\min f)\|\nabla u\|, & x \in E, \quad t>0 \\ u(x, 0)=f(x), & x \in E\end{cases}
$$

This couple of PDEs are particular cases of the Hamilton-Jacobi models discussed in Section 4. More precisely, it corresponds to the case of the Hamiltonians given in expressions (78) and (79). We can therefore conclude that the solution $u(x, t)$ for + sign of model (112) is equivalent to viscous dilation $\delta_{\wedge}^{\mathrm{visc}}(f)$, but for - sign it is not exactly equivalent to the viscous erosion $\varepsilon_{\wedge}^{\text {visc }}(f)$. In our terminology, the latter is a case of adjoint infmax convolution while the solution for - sign is an infmax convolution with the complemented structuring function. Similarly, solution $u(x, t)$ of model (113) is equivalent to viscous erosion $\varepsilon_{\vee}^{\mathrm{visc}}(f)$ for $-\operatorname{sign}$ and once again, for + sign a supmin convolution is obtained.

As a conclusion of this part on viscous operators, let us clarify that when using our formulation of such operators it becomes evident that viscous openings and closings do not require any implementation using upper level set decompositions and then stacking. They can be implemented straightforward as ( $\max , \min )$-convolutions, which notably reduces the computation load.

\subsection{Links with Boolean random function characterization}

There is a theory of random set modelling intimately related to morphological image processing. This theory was introduced by Matheron [46] and yields a sound family of probabilistic models for microstructures (dealing with variability, heterogeneity, multi-scaling, etc.). It has been widely used for modeling and predicting the average macroscopic response of random media from their microstructure. Morphological operators are used to test and to select appropriate models (i.e., estimate their parameters) from images and to simulate new images following the model. The set theory was later generalized to random functions. Our 
aim here is just to point out how (max, min)-convolutions are related to random function characterization in the framework of this theory.

Boolean random set characterization. First of all, we briefly recall the framework for Boolean random sets. Let us assume that we work in the Euclidean space $\mathbb{R}^{n}$. A Boolean Random Closed Set (or Boolean RACS) $X \subset \mathbb{R}^{n}$ of parameters $\left(\theta, A^{\prime}\right)$ is obtained as follows [46]. Consider, on the one hand, a realization of a Poisson point process of intensity $\theta \geq 0$; i.e., the Poisson points $x_{i}$ are the germs. It means that the number of points in a borel set $B$, denoted $N(B)$, follows a Poisson distribution of parameter $\theta \mu_{n}(B)$ (i.e., mean proportional to the Lebesgue measure of $B)$ :

$$
P\{N(B)=k\}=e^{-\theta \mu_{n}(B)} \frac{\left(\theta \mu_{n}(B)\right)^{k}}{k !} .
$$

Consider, on the other hand, a family of compact closed random sets $A^{\prime}$ i.i.d. located at the origin; i.e., $A^{\prime}$ is the primary grain. Then, implant at each germ a realization of the primary grain and take the union for all the points; i.e.,

$$
X=\bigcup_{x_{i}} A_{x_{i}}^{\prime}
$$

Any shape can be used for $A^{\prime}$ : convex or non convex, connected or not connected.

Given a RACS $X$, let us introduce the Choquet capacity $T_{X}(K)$, defined on the compact sets $K \in \mathcal{K}$ as the probability that the compact $K$ hits the set $X$, i.e.,

$$
T_{X}(K)=P\{K \cap X \neq \emptyset\}=1-P\left\{K \subset X^{c}\right\}=1-Q_{X}(K)
$$

The functional $T$ has the following properties: (i) $T$ is bounded with $0 \leq T \leq 1$ and $T(\emptyset)=0$; (ii) $T$ is increasing in the sense $T(K) \leq T\left(K \cup K^{\prime}\right), K, K^{\prime} \in \mathcal{K}$; (iii) $T$ is upper-semicontinuous. In fact, $T$ acts as a distribution function of a random variable. If $X$ is a stationary RACS, then its Choquet capacity is shift invariant, i.e., $T_{X}\left(K_{h}\right)=T_{X}(K)$. There is a fundamental result, named the Choquet-Matheron-Kendall theorem, saying that a RACS is characterized (described completely) by the probabilities $Q_{X}(K)$ as $K$ spans the class of the compact sets $\mathcal{K}$. From a practical viewpoint, it is important to note that the experimental estimation of $T_{X}(K)$ and $Q_{X}(K)$ from images is done using realizations of $K$ and morphological transforms; e.g., dilation or erosion:

$$
\begin{aligned}
T_{X}\left(K_{x}\right) & =P\left\{K_{x} \cap X \neq \emptyset\right\}=P\{x \in X \oplus \check{K}\} ; \\
Q_{X}\left(K_{x}\right) & =P\left\{K_{x} \subset X^{c}\right\}=P\left\{x \in X^{c} \ominus K\right\} .
\end{aligned}
$$

These expressions can be simplified in the case of a Boolean RACS. Let $X$ be a Boolean RACS of Poisson density $\theta$ and primary grain $A^{\prime}$. The Choquet capacity of $X$ for any $K \in \mathcal{K}$ is given by [46]:

$$
T_{X}(K)=1-Q_{X}(K)=1-e^{-\theta \mathbb{E}\left\{\mu_{n}\left(A^{\prime} \oplus \check{K}\right)\right\}} .
$$


Thus the number of primary grains hit by $K$ follows a Poisson distribution with average $\theta \mathbb{E}\left\{\mu_{n}\left(A^{\prime} \oplus \check{K}\right)\right\}$. Now taking different $K$, such as a singleton $K=\{x\}$, a couple of points $K=\{x, x+d\}$, etc. the moments of $Q(K)$ are obtained. The empirical values are used to fit the parameters of the model. In addition, if the primary grain is a convex set, using Steiner formula, the expressions of $\mathbb{E}\left\{\mu_{n}\left(A^{\prime} \oplus \lambda \check{K}\right)\right\}$ are polynomials in $\lambda$. These computations are used to test the validity of the Boolean model assumption.

Choquet capacity of random functions. The Boolean random functions are a generalization of the Boolean RACS. The basic idea of Boolean random functions was introduced by Jeulin [31] for modeling rough surfaces; this model has been extensively studied and generalized by Serra [60] and by Préteux and Schmitt [54]. We follow here the results formulated by Jeulin [32]; for a more recent development, see [33].

The functional framework involves to generalize the RACS $X$ by means of an upper semicontinuous function $Z(x)$ and the compact test set $K$ by a lower semicontinuous test function $\tau(x)$. Then, as for the case of random sets, the basic mathematical tool to deal with random functions is the Choquet capacity.

Let $Z(x): \mathbb{R}^{n} \rightarrow \mathbb{R} \cup\{-\infty\}$ be an USC random function, almost surely bounded and with compact (closed) upper level sets. The test function $\tau(x): K \rightarrow \mathbb{R} \cup\{+\infty\}$ is defined on a compact support $K \in \mathcal{K}$ and it is a LSC. The Choquet capacity $T_{Z}(\tau)$ defined over the set of test functions is now given by

$$
T_{Z}(\tau)=P\left\{x \in D_{Z}(\tau)\right\}=1-Q_{Z}(\tau)=1-P\left\{x \in\left[D_{Z}(\tau)\right]^{c}\right\},
$$

where $D_{Z}(\tau)$ is the set

$$
D_{Z}(\tau)=\left\{\exists y \in \mathbb{R}^{n}: Z(y) \geq \tau(x-y)\right\}
$$

such that the complementary of the event $D_{Z}(\tau)$ is given by the following random set

$$
\left[D_{Z}(\tau)\right]^{c}=\{x: Z(x+y)<\tau(y), \forall y \in K\} .
$$

By convention it is considered that $\tau(x)=+\infty$ for $x \notin K$. Thus the functional $T_{Z}(\tau)$ gives the probability that the deterministic function $\tau$ "hits" the hypograph of the random function $Z$, which is a closed set. We should notice that by the lower semicontinuity of $\tau$, its epigraph is a closed set.

For the points $x$ which satisfy $Z(x+y)<\tau(y)$, it holds: $Y_{h}^{-}(\tau) \subset\left[X_{h}^{+}\left(Z_{x}\right)\right]^{c}, \forall h \in \mathbb{R}$. Thus one gets

$$
\begin{aligned}
{\left[D_{Z}(\tau)\right]^{c} } & =\left\{x: \forall h \in \mathbb{R}, x \in\left[X_{h}^{+}\left(Z_{x}\right)\right]^{c} \ominus Y_{h}^{-}(\tau)\right\} \\
& =\bigcap_{h \in \mathbb{R}} Y_{h}^{-}\left(Z_{x}\right) \ominus Y_{h}^{-}(\tau) .
\end{aligned}
$$


The dual by complement is given by

$$
D_{Z}(\tau)=\bigcup_{h \in \mathbb{R}} X_{h}^{+}\left(Z_{x}\right) \oplus \check{Y}_{h}^{-}(\tau) .
$$

As for the case of random sets, we are only interested in stationary functions; i.e., $T_{Z}(\tau)$ is invariant to translation of $\tau$.

In order to relate these functionals with ( $\max , \min )$-convolutions, we only need to remind from (37) that

$$
\left(f \nabla^{*} b\right)(x)=\sup \left\{h \in \overline{\mathbb{R}}: X_{h}^{+}(f) \oplus Y_{h}^{-}\left(b^{c}\right)\right\} .
$$

Thus we can now write the Choquet capacity $T_{Z}(\tau)$ using the adjoint supmin convolution:

$$
T_{Z}(\tau)=P\left\{x \in \bigcup_{h \in \mathbb{R}} X_{h}^{+}(Z) \oplus \check{X}_{h}^{-}(\tau)\right\}=P\left\{x \in \bigcup_{h \in \mathbb{R}} X_{h}^{+}\left(Z \nabla^{*} \check{\tau}^{c}\right)\right\} .
$$

In addition, if we assume that the random function $Z$ is a nonnegative function, and we denote by $[Z]_{+}$the support set of $Z(x)>0$, then we can write

$$
T_{Z}(\tau)=P\left\{x \in\left[Z \nabla^{*} \check{\tau}^{c}\right]_{+}\right\}
$$

It is not surprising to discover that the dilation of $X$ by $K$ in the RACS Choquet capacity is generalized to the adjoint supmin of $Z$ by the $\tau$ in the functional Choquet capacity: we only need to note how the adjoint supmin is defined. The complement of $\tau$ is due to the definition of $\nabla^{*}$ as the adjoint of $\triangle$.

Boolean random functions. The particular case of the Boolean random functions naturally involves the construction of a random function over a Poisson point process. We focus on the case of the so-called Boolean islands [60]. Namely, three steps are needed.

1. A Poisson point process in $\mathbb{R}^{n}$ of intensity $\theta$.

2. At each point of the process $x_{i}$, we set up a nonnegative random function $f_{i}(x)$, called the primary random function, drawn independently from a distribution $f^{\prime}$; i.e., $f_{i} \sim f^{\prime}$.

3. The Boolean random function $Z(x)$ is obtained as

$$
Z(x)=\bigvee_{x_{i}} f_{i}\left(x-x_{i}\right)
$$

Obviously, the set $X_{h}^{+}(Z)$ is a Boolean random set with primary grain $A^{\prime} \sim X_{h}^{+}\left(f^{\prime}\right)$.

In addition, in the case of the Boolean random function, one has:

$$
D_{Z}(\tau)=\bigcup_{x_{i}}\left[D_{f_{i}}(\tau)\right]_{x_{i}} \Rightarrow\left[Z \nabla^{*} \check{\tau}^{c}\right]_{+}=\left[\bigvee_{x_{i}} f_{i}\left(x-x_{i}\right) \nabla^{*} \check{\tau}^{c}\right]_{+},
$$


such that $D_{Z}(\tau)$ is a Boolean RACS with the primary grain $D_{f^{\prime}}(\tau)=\left[f^{\prime} \nabla^{*} \check{\tau}^{c}\right]_{+}$. This property is very useful in practice for the identification of a model of Boolean random function using tools for Boolean RACS models. The expression of the Choquet capacity for a Boolean random function has also a nice expression.

Proposition 23 Given a Boolean random function of parameters $\left(\theta, f^{\prime}\right)$, its Choquet capacity is given by [32]:

$$
1-T_{Z}(\tau)=Q_{Z}(\tau)=\exp \left(-\theta \mathbb{E}\left\{\mu_{n}\left(\left[f^{\prime} \nabla^{*} \check{\tau}^{c}\right]_{+}\right)\right\}\right),
$$

and therefore, the logarithm of the characteristic functional is given by

$$
\log Q_{Z}(\tau)=-\theta \mathbb{E}\left\{\mu_{n}\left(\left[f^{\prime} \nabla^{*} \check{\tau}^{c}\right]_{+}\right)\right\} .
$$

Proof. This proof is inspired from the one of the Boolean model provided in [38]. Let us denote by $X(B)$ the union of the primary grains implanted in a bounded domain $B$. The number of primary grains follows a Poisson distribution of parameter $\theta \mu_{d}(B)$. Take an uniform point $x \in B$ and implant a primary function at $x$, denoted $f_{x}^{\prime}$. It is "disjoint" with $\tau$ with probability

$$
P\left\{x \notin D_{f^{\prime}}(\tau)\right\}=1-\frac{\mathbb{E}\left\{\mu_{d}\left(D_{f^{\prime}}(\tau) \cap B\right)\right\}}{\mu_{d}(B)} .
$$

When the number of points equals $i$, the $i$ primary functions has been independently and uniformly implanted. Consequently,

$$
Q_{Z}^{B}(\tau)=\sum_{i=0}^{+\infty} e^{-\theta \mu_{d}(B)} \frac{\left(\theta \mu_{d}(B)\right)^{i}}{i !}\left(1-\frac{\mathbb{E}\left\{\mu_{d}\left(D_{f^{\prime}}(\tau) \cap B\right)\right\}}{\mu_{d}(B)} .\right)^{i}
$$

This sum is equal to

$$
Q_{Z}^{B}(\tau)=e^{-\theta \mathbb{E}\left\{\mu_{d}\left(D_{f^{\prime}}(\tau) \cap B\right)\right\}}
$$

The results should be extended to complete domain of definition of $Z$. Let us consider an increasing sequence $B_{n}$ of bounded domains in $\mathbb{R}^{d}$. The events " $K \cap X\left(B_{n}\right)=\emptyset$ " of probability $Q_{Z}^{n}(K)$ are an increasing sequence of intersection " $K \cap X=\emptyset$ ". By the axiom of continuity we have

$$
Q_{Z}(K)=\lim _{n \rightarrow+\infty} Q_{Z}^{n}(K)=\lim _{n \rightarrow+\infty} e^{-\theta E\left\{\mu_{d}\left(D_{f^{\prime}}(\tau) \cap B_{n}\right)\right\}}=e^{-\theta \mathbb{E}\left\{\mu_{d}\left(D_{f^{\prime}}(\tau)\right)\right\}} .
$$

Finally, from (117), one have

$$
D_{f^{\prime}}(\tau)=\left[f^{\prime} \nabla^{*} \check{\tau}^{c}\right]_{+}
$$

From the expression (119), different particular cases $\tau$ can be used to obtain for instance the spatial law of the Boolean random function, its bivariate distribution, etc. [32]. 
A particular case corresponds to the case of a test function of type inverted cylinder of height $k \in \mathbb{R}$ and base $K$ :

$$
\tau(x)=\{K ; k\}=\left\{\begin{array}{cll}
k & \text { if } & x \in K \\
+\infty & \text { if } & x \notin K
\end{array}\right.
$$

such that one has

$$
\left[D_{Z}(\tau)\right]^{c}=\left\{x: \bigvee_{y \in K} Z(x+y)<k\right\} .
$$

This expression can be rewritten in morphological terms as

$$
\bigvee_{y \in K} Z(y)<k \Leftrightarrow K_{x} \subset Y_{k}^{-}(Z) \Leftrightarrow x \in\left(Y_{k}^{-}(Z) \ominus K\right),
$$

and the complementary event

$$
\bigvee_{y \in K} Z(y) \geq k \Leftrightarrow x \in\left[Y_{k}^{-}(Z) \ominus K\right]^{c}=X_{k}^{+}(Z) \oplus \check{K}=D_{Z}(\tau)
$$

For this type of test function $\{K ; k\}$, we have in the Boolean case

$$
P\left\{\bigvee_{x \in K} Z(x)<k\right\}=\exp \left(-\theta \mathbb{E}\left\{\mu_{n}\left(X_{k}^{+}\left(f^{\prime}\right) \oplus \check{K}\right)\right\}\right)
$$

It gives the probability distribution of the Boolean random function $Z(x)$ after a change of support by supremum over the compact set $K$.

We believe that the interest of the expression (119) of $Q_{Z}(\tau)$, based on a (max, min)convolution, opens avenues regarding a more widely use of Boolean random functions. In particular, we can see how the role of convexity of the primary grain $A^{\prime}$ in the Boolean model is replaced here by the convexity of the upper level sets of the primary function $f^{\prime}$, which involves that the most interesting case corresponds to quasiconcave primary functions $f^{\prime}$. Other issues, as the interest of the complement of the conic structuring function $c_{\alpha, \lambda}^{c}(x)=\lambda^{-1}\|x\|+\alpha$ as privileged test function, as well as the simulation of Boolean random function using the Hamilton-Jacobi PDEs discussed above, will be considered in ongoing research.

Bibliographic remark. In the initial theory of Boolean random functions, it was classically considered as involving the Minkowski addition of the subgraph of function $Z(x)$ by compact test sets $K$, where $K$ is seen as a subset of $\mathbb{R}^{n} \times \mathbb{R}[60,54]$, which is not equivalent to the use of structuring functions. The formulation based on test functions and level-set representation of the Choquet capacity introduced by Jeulin [32] has allowed us to link Boolean random function characterization with adjoint supmin convolution.

We believe that most results proved by Goutsias [28] for the discrete framework of RACS can be now extended to discrete random function. 
Let us note that if we consider the case of Boolean functions defined in the unit interval $[0,1]$, the immediate consequence is the fact that the underlaying framework can be seen as the appropriate one to model Boolean fuzzy sets. Remember that the key ingredient is the implication of the Gödel-Brower Logic. Up to the best of our knowledge, no previous work has ever studied the interest of fuzzy morphological operators to model Boolean fuzzy sets. This point seems now natural and deserves a deeper effort of research.

Concerning just the theory of random fuzzy closed sets, some efforts have been paid recently in order to generalize Matheron's results for RACS to fuzzy sets. In particular, there are already some relevant results including Choquet theorem for random USC functions based on Lawson topology in the continuous lattice theory, that includes also some tentative of generalization of Matheron's hit-or-miss topology. However many topological questions, including measurability considerations, are still open [51,69]. In this context, we think that the Lawson topology of continuous lattices is also relevant for the study of the topological properties of (max, min)-convolutions.

\subsection{Links with geodesic dilation and erosion}

In Section 2, on the background on classical morphological operators, we did not consider the notion of geodesic dilation and erosion [37, 66]. Geodesic operators are extremely useful transforms for solving many image processing tasks [66, 62].

The philosophy of these operators is rather different of the classical convolution-like dilation and erosion, since the role of the structuring element/function is replaced by an external function called the reference (or geodesic mask). More precisely, the elementary geodesic dilation $\delta_{X}^{1-g e o}(Y)$ of a set $Y$ included in the geodesic mask $X, X, Y \subset \mathbb{R}^{n}$, is defined as

$$
\delta_{X}^{1-g e o}(Y)=(Y \oplus B) \cap X,
$$

where $B$ is the unit Euclidean ball. The elementary geodesic erosion $\varepsilon_{X}^{1-g e o}(Y)$ is defined by set complementation:

$$
\varepsilon_{X}^{1-g e o}(Y)=X \backslash \delta_{X}^{1-g e o}(X \backslash Y)=\left(\left(Y \cup X^{c}\right) \ominus B\right) \cap X .
$$

We note that geodesic dilation and erosion for sets are dual both by complement and by adjunction. Larger operations are obtained by iteration and keeping $X$ fixed. Thus, the geodesic dilation of size $n$ of $Y$ corresponds to the space swept by the geodesic balls $B_{X}(x, n)$ of size $n$ whose center $y$ is included in $Y$; the geodesic erosion of size $n$ corresponds to the centers of the geodesic balls of size $n$ of $X$ included in $Y$ [37], i.e.,

$$
\begin{aligned}
& \delta_{X}^{n-g e o}(Y)=\left\{x \in X: B_{X}(x, n) \cap Y \neq \emptyset\right\}=\bigcup_{y \in Y} B_{X}(y, n), \\
& \varepsilon_{X}^{n-g e o}(Y)=\left\{x \in X: B_{X}(x, n) \subset Y\right\} .
\end{aligned}
$$


Extension from the set operators to the geodesic operators for functions is based on a level set based transposition of set geodesic dilation, which leads to the geodesic dilation $\delta_{g}^{1-g e o}(f)$ of a function $f$ in the geodesic space of $g$ (or under $g$, since it is assumed that $f \leq g$ ):

$$
\delta_{g}^{1-g e o}(f)=(f \oplus B) \wedge g, \quad f \leq g .
$$

The geometric interpretation is similar to the one for the set case, by considering the notion of sweeping geodesic cylinders. Then, the geodesic erosion is classically defined using the duality by complement from (122) to obtain [66]:

$$
\varepsilon_{g}^{1-g e o}(f)=(f \ominus B) \vee g, f \geq g .
$$

In fact, it is easy to see that the functional geodesic erosion (123) is not the operator obtained by the level set extension of the set geodesic erosion (121). That involves in fact that $\varepsilon_{g}^{1-g e o}(f)$ and $\delta_{g}^{1-g e o}(f)$ do not form an adjunction. It is very surprising for us that the study of the geodesic adjunction has been the object of depth research only in a relatively recent and illuminating study by Beucher [13].

Starting from the dual by complement operators (122) and (122), computation of the corresponding adjoint operators is provided in detail in [13]. The four elementary geodesic operators are given by

$$
\begin{aligned}
& g \leq f, \quad \bar{\varepsilon}_{g}^{1-g e o}(f)=(f \ominus B) \vee g \stackrel{\text { adjoint }}{\longleftrightarrow} \bar{\delta}_{g}^{1-g e o}(f)=[(f \wedge m) \oplus B] \vee g \\
& \text { with } m=\{x: f>g\} \\
& \uparrow \text { dual } \\
& \uparrow \text { dual } \\
& g \geq f, \quad \underline{\delta}_{g}^{1-g e o}(f)=(f \oplus B) \wedge g \stackrel{\text { adjoint }}{\longleftrightarrow} \underline{\varepsilon}_{g}^{1-g e o}(f)=[(f \vee m) \ominus B] \wedge g \\
& \text { with } m=\{x: f=g\}
\end{aligned}
$$

In order to relate geodesic operators to (max, min) algebra, we can rewrite the geodesic dilation $\underline{\delta}_{g}^{1-g e o}(f)$ using the distributivity:

$$
\underline{\delta}_{g}^{1-g e o}(f)(x)=(f \oplus B)(x) \wedge g(x)=g(x) \wedge \sup _{z \in B(x)} f(x-z)=\sup _{z \in B(x)}\{g(x) \wedge f(x-z)\} .
$$

We can also find an alternative expression for its adjoint geodesic erosion $\underline{\delta}_{g}^{1-g e o}(f)$, with $f \leq g$ :

$$
\underline{\varepsilon}_{g}^{1-g e o}(f)(x)=[(f \vee m) \ominus B](x) \wedge g(x)=\inf _{z \in B(x)}\{g(x) \wedge(f(x-z) \vee m(x-z))\} .
$$

First, we note that using the mask function given by $m(x)=\top$ if $f(x)=g(x)$ and $m(x)=\perp$ if $f(x)<g(x)$, one has $(f \vee m)(x)=\top$ if $f(x)=g(x)$ and $(f \vee m)(x)=f(x)$ if $f(x)<g(x)$. Second, we introduce the operator $\wedge^{*-g e o}$ as

$$
g(x) \wedge^{*-g e o} f(x-z)=g(x) \wedge(f(x-z) \vee m(x-z))=\left\{\begin{array}{cl}
g(x) & \text { if } f(x-z)=g(x-z) \\
f(x-z) \wedge g(x) & \text { if } f(x-z)<g(x-z)
\end{array}\right.
$$


Thus, we finally obtain

$$
\underline{\varepsilon}_{g}^{1-g e o}(f)(x)=\inf _{z \in B(x)}\left\{g(x) \wedge^{*-g e o} f(x-z)\right\} .
$$

The operator $\wedge^{*-g e o}$ can be seen as the "geodesic counterpart" of the adjoint operator to the minimum (24). Going further with the parallelism, we believe that, even if the geodesic dilation and its adjoint geodesic erosion are not convolution-like operators, it is seems clear from expressions (124) and (125) that these two operators are structurally similar respectively to the supmin convolution(20) and the adjoint infmax convolution (22). An equivalent interpretation is straightforward obtained for the geodesic erosion $\bar{\varepsilon}_{g}^{1-g e o}(f)$ and its adjoint geodesic dilation $\bar{\delta}_{g}^{1-\text { geo }}(f)$.

Thus, we would like to conclude by stating that geodesic dilation and erosion are naturally defined as operators in ( $\max , \min )$-algebra. We plan to fully exploit this new viewpoint in future research, including links of geodesic operators with nonlinear analysis and HamiltonJacobi PDEs.

\section{Conclusion and Perspectives}

Operators and filters underlying a formulation as ( $\max , \min )$-convolutions are common in the state-of-the-art of mathematical morphology. However, their study per se has been neglected. From this epistemological viewpoint, we can conclude that the role of ( $\max , \min$ )-convolutions has been somewhat overshadowed by a multiplicity of viewpoints (fuzzy, viscous, "hitting of functions" in Choquet capacity, etc.)

In order to address this theoretical lack, we have developed in our paper a rigorous formulation and characterization of the four convolution-like operators in (max, min)-algebra.

Just concerning the need of specifically four morphological operators in such algebra, we point out that this is clearly justified by the fact that one deals with two dualities, by complement and by adjunction. This principle is not exclusive from mathematical morphology, in optimization theory involving minimum or maximum operations, it is also a natural principle well formalized [24].

We have proven that (max, min)-openings are compatible with Matheron's axiomatic of Euclidean granulometries for functions with quasiconcave structuring functions. This was not the case for $(\max ,+)$-openings. We have also shown that the adjoint supmin convolution is the operator underlying the extension of Matheron's characterization of Boolean random closed sets to the case of Boolean random upper semicontinuous function.

For all these reasons, we strongly think that ( $\max , \min$ )-convolution provides the natural framework to generalize some key notions of Matheron's theory from sets to functions.

All the theoretical results provided in the paper are formulated in a continuous setting. Although it may seem that the transition from the continuous to the discrete case is straight- 
forward (this is usually the case in the theory of mathematical morphology), this transition is often challenging with a certain number of notions, such as convexity. We plan to revisit all our results in a fully discrete framework.

All the algebraic results (and most of nonlinear analysis ones) on (max, min)-convolutions considered here are valid for functions supported in a general Banach space, consequently more general that the Euclidean space $\mathbb{R}^{n}$. In this generalization context, we plan to consider in particular the case of ( $\max , \min )$-morphology for real-valued images on Riemannian manifolds. It will be a parallel study the our recent work on the extension of $(\max ,+)$-morphology to Riemannian images [6].

A final remark concerning the generalization of the morphological convolutions proposed here. If we compare for instance the classical erosion and the infmax erosion:

$$
\begin{aligned}
& (f \ominus b)(x)=\inf _{y}\left\{f(y)+\check{b}^{c}(x-y)\right\}, \\
& (f \triangle b)(x)=\inf _{y}\left\{f(y) \vee \check{b}^{c}(x-y)\right\},
\end{aligned}
$$

they can be considered as a particular case of the following transformation:

$$
\left(f(\bar{\wedge})_{p} b\right)(x)=\inf _{y}\left\{N_{p}\left(f(y), \check{b}^{c}(x-y)\right)\right\}
$$

where $N_{p}(r, s)$ is a monotone norm, for instance the $L^{p}$ Minkowski norm; i.e., for $p \geq 1$

$$
N_{p}=\left(|r|^{p}+|s|^{p}\right)^{\frac{1}{p}}
$$

such that $(f \ominus b)=\left(f(\bar{\wedge})_{1} b\right)$ and $(f \triangle b)=\left(f(\bar{\wedge})_{\infty} b\right)$. Besides these limit cases, the study of the properties of the operator (126) and its dual and adjoint counterparts is potentially relevant in mathematical morphology theory. Some results already obtained in the domain of convex analysis, see [67], can be a good starting point.

In previous work, we have considered a nonlinearization of continuous diffusion [3] and bilateral discrete filtering [4] in order to produce families of processed images from (Gaussian) convolution in $(+, \times)$-algebra to (parabolic) morphological operators in $(\max ,+)$-algebra. The development of a computational framework which unifies the three algebras, from (Gaussian) convolution in $(+, \times)$-algebra to (conic) morphological operators in ( $\max , \min )$-algebra would be very powerful for the efficient implementation of generic convolution in specific hardware/software architectures.

Concerning the Hamilton-Jacobi PDEs associated to supmin and infmax convolutions, we have not discussed their numerical solution. Numerical algorithms for Hamilton-Jacobi PDE are relatively well known, in particular the Osher and Sethian algorithm [52] or the less diffusive Rouy and Tourin schema [56]. Alternative schemas can be also found in [43, 16, 21]. 
Nevertheless, ongoing research on the behavior of such numerical approaches is needed to state the accuracy, convergence, etc. for different structuring functions, i.e., different Hamiltonians. As usual, the interest of PDE approaches is to provide infinitesimal computation with a geometrically better approximation of the Euclidean distance. In addition, in the case of (max, min)-convolutions framework, it is easy to implement many different filtering effects only by changing the Hamiltonian. On the contrary, we should notice that the PDE approach is not appropriate for implementing advanced operators; i.e., alternate sequential filters obtained by the composition of openings and closings at various scales.

We sincerely hope that results reviewed in Sections 4 and 5 will help a general reader to appreciate the fact that applied mathematics underlying morphological operators are well founded and pertinent from nonlinear analysis. Theoretical roots of mathematical morphology are nowadays formulated in the framework of algebra (more specifically, using complete lattice theory): we expect that the results provided in the paper justify a complementary coherent theoretical viewpoint of morphological operators from nonlinear analysis.

Acknowledgement. I would like to thank F. Meyer for fruitful discussions on this work. Many thanks also to D. Jeulin for his precisions on Boolean random function characterization.

\section{References}

[1] L. Alvarez, F. Guichard, P-L. Lions, J-M. Morel. Axioms and fundamental equations of image processing. Arch. for Rational Mechanics, Vol. 123, No. 3: 199-257, 1993.

[2] O. Alvarez, E.N. Barron, H. Ishii. Hopf-lax formulas for semicontinuous data. Indiana Univ. Math. J., Vol. 48, 993-1035, 1999.

[3] J. Angulo. Generalised Morphological Image Diffusion. HAL preprint, hal-00789162, 2013.

[4] J. Angulo. Morphological Bilateral Filtering. SIAM Journal on Imaging Sciences, Vol. 6, No. 3, 1790-1822, 2013.

[5] J. Angulo, S. Velasco-Forero. Stochastic Morphological Filtering and Bellman-Maslov Chains. In Proc. of ISMM'13 (11th International Symposium on Mathematical Morphology), LNCS 7883, Springer-Verlag, p. 171-182, 2013.

[6] J. Angulo, S. Velasco-Forero. Riemannian Mathematical Morphology. Pattern Recognition Letters, Vol. 47, 93-101, 2014.

[7] A.B. Arehart, L. Vincent, B.B. Kimia. Mathematical morphology: The Hamilton-Jacobi connection. In Proc. of IEEE 4th Inter. Conf. on Computer Vision (ICCV'93), 215-219, 1993. 
[8] D. Attouch, D. Aze. Approximation and regularization of arbitray functions in Hilbert spaces by the Lasry-Lions method. Annales de l'I.H.P., section C, Vol. 10, No. 3, 289$312,1993$.

[9] M. Avriel, W.E. Diewert, S. Schaible, I. Zang. Generalized Concavity, Plenum Press, 1988.

[10] M. Bardi, L.C. Evans. On Hopf's formulas for solutions of Hamilton- Jacobi equations. Nonlinear Analysis, Theory, Methods and Applications, Vol. 8, No. 11, 1373-1381, 1984.

[11] E.N. Barron, R. Jensen, W. Liu. Hopf-Lax formula for $u_{t}=H(u, D u)=0$. J. Differential Equations, Vol. 126, 48-61, 1996.

[12] E.N. Barron, R. Jensen, W. Liu. Hopf-Lax formula for $u_{t}=H(u, D u)=0$, II. Comm. Partial Differential Equations, Vol. 22, 1141-1160, 1997.

[13] S. Beucher. About a problem of definition of the geodesic erosion. CMM/Mines ParisTech Technical Report, 16 p., 2011.

http://cmm.ensmp.fr/ beucher/publi/GeodesicErode_eng.pdf

[14] I. Bloch. Duality vs. adjuntion for fuzzy mathematical morphology and general form of fuzzy erosions and dilations. Fuzzy Sets and Systems, Vol. 160, 1858-1867, 2009.

[15] R. van den Boomgaard, L. Dorst. The morphological equivalent of Gaussian scale-space. In Proc. of Gaussian Scale-Space Theory, 203-220, Kluwer, 1997.

[16] M. Breuß, J. Weickert. Highly accurate PDE-based morphology for general structuring elements. In Proc. of Scale Space and Variational Methods in Computer Vision, LNCS Vol. 5567, 758-769, Springer, Berlin, 2009.

[17] R.W. Brockett, P. Maragos. Evolution equations for continuous-scale morphology. IEEE Trans. on Signal Processing, Vol. 42, No. 12, 3377-3386, 1994.

[18] F.H. Clarke, R.J. Stern (Eds.). Nonlinear Analysis, Differential Equations and Control. NATO Science Series C: Mathematical and Physical Sciences, Vol. 528, Kluwer, 1999.

[19] M.G. Crandall, H. Ishii, P.-L. Lions. User's guide to viscosity solutions of second order partial differential equations. Bulletin of the American Mathematical Society, Vol. 27, No. 1:1-67, 1992.

[20] T.-Q. Deng, H.J.A.M. Heijmans. Grey-Scale Morphology Based on Fuzzy Logic. Journal of Mathematical Imaging and Vision, Vol. 16, No. 2, 155-171, 2002.

[21] E.H.S. Diop, J. Angulo. Multiscale Image Analysis Based on Robust and Adaptive Morphological Scale-Spaces. HAL preprint, hal-00975728, 2014. 
[22] L. Dorst, R. van den Boomgaard. Morphological Signal Processing and the Slope Transform. Signal Processing, Vol. 38, 79-98, 1994.

[23] E.A. Engbers, R. Van Den Boomgaard, A.W.M. Smeulders. Decomposition of Separable Concave Structuring Functions. Journal of Mathematical Imaging and Vision, Vol. 15, No. 3, 181-195, 2001.

[24] J. Flachs, M.A. Pollatschek. Duality theorems for certain programs involving minimum or maximum operations. Mathematical Programming, Vol. 16, 348-370, 1979.

[25] G. Gierz, K.H. Hofmann, K. Keimel, J.D. Lawson, M.Mislove, D.S. Scott. A Compendium of Continuous Lattices. Springer-Verlag, Berlin, 1980.

[26] M. Gondran. Analyse MINMAX. C.R. Acad. Sci. Paris, t. 323, Série I, 1249-1252, 1996.

[27] M. Gondran, M. Minoux. Graphs, Dioids and Semirings: New Models and Algorithms. Springer, 2008.

[28] J. Goutsias. Morphological analysis of discrete random shapes. Journal of Mathematical Imaging and Vision, Vol. 2, 193-215, 1992.

[29] H.J.A.M. Heijmans. Morphological image operators. Academic Press, Boston, 1994.

[30] P.T. Jackway, M. Deriche. Scale-Space Properties of the Multiscale Morphological Dilation-Erosion. IEEE Trans. Pattern Anal. Mach. Intell., 18(1): 38-51, 1996.

[31] D. Jeulin, P. Jeulin. Synthesis of Rough Surfaces by Random Morphological Model. Proc. of 3rd European Symposium of Stereology, Stereol. Iugosl., Vol. 3, Suppl. 1, 239 246, 1981.

[32] D. Jeulin. Modèles Morphologiques de Structures Aléatoires et de Changement d'Echelle. Thèse d'Etat ès Sciences Physiques, Université de Caen, France, 1991.

[33] D. Jeulin. Boolean Random Functions. In (V. Schmidt, Ed.) Stochastic Geometry, Spatial Statistics and Random Fields, Chapter 5. Series: Lecture Notes in Mathematics, Vol. 2120, Springer 2014.

[34] V. Kolokoltsov, V.P. Maslov. Idempotent Analysis and Its Applications, Springer 1997.

[35] E.J. Kraus, H.J.A.M. Heijmans, E.R. Dougherty. Gray-scale granulometries compatible with spatial scalings. Signal Processing, Vol. 34, No. 1, 1-17, 1993.

[36] J.M. Lasry, P.-L. Lions. A remark on regularization in Hilbert spaces. Israel Journal of Mathematics, Vol. 55, 257-266, 1986. 
[37] Ch. Lantuéjoul, S. Beucher. On the use of the geodesic metric in image analysis. Journal of Microscopy, Vol. 121, Part 1, 39-49, 1981.

[38] Ch. Lantuéjoul. Cours "Les ensembles aléatoires", (Lecture notes). Ecole des Mines de Paris, 1993.

cg.ensmp.fr/bibliotheque/public/LANTUEJOUL_Cours_00578.pdf

[39] D.T. Luc, M. Volle. Levels sets Infimal Convolution and Level Addition. Journal of Optimization Theory and Applications, Vol. 94, No. 3, 695-714, 1997.

[40] P. Maragos, R.W. Schafer. Morphological filters-Part I: Their set-theoretic analysis and relations to linear shift-invariant filters. IEEE Trans. on Acoustics, Speech and Signal Processing, Vol.35, No. 8, 1153-1169, 1987.

[41] P. Maragos. Slope Transforms: Theory and Application to Nonlinear Signal Processing. IEEE Trans. on Signal Processing, Vol. 43, No. 4, 864-877, 1995.

[42] P. Maragos. Differential morphology and image processing. IEEE Transactions on Image Processing, Vol. 5, No. 1: 922-937, 1996.

[43] P. Maragos. Algebraic and PDE Approaches for Lattice Scale-Spaces with Global Constraints. International Journal of Computer Vision, Vol. 52, Issue 2/3, 121-137, 2003.

[44] P. Maragos. Lattice image processing: a unification of morphological and fuzzy algebraic systems. Journal of Mathematical Imaging and Vision, Vol. 22, No. 2-3, 333-353, 2005.

[45] P. Maragos, C. Vachier. A PDE Formulation for Viscous Morphological Operators with Extensions to Intensity- Adaptive Operators. In Proc. of 15th IEEE International Conference on Image Processing (ICIP'08), pp. 2200-2203, 2008.

[46] G. Matheron. Random Sets and Integral Geometry. Wiley, New York, 1975.

[47] F. Meyer. Inondation par des fluides visqueux. CMM/Mines ParisTech Technical Report, 1993.

[48] F. Meyer. Operateurs morphologiques visqueux. CMM/Mines ParisTech Technical Report, 2008.

[49] J.J. Moreau. Inf-convolution, convexité des fonctions numériques. J. Math. Pures Appl., Vol. 49, 109-154, 1970.

[50] M. Nachtegael, E.E. Kerre. Connections between binary, gray-scale and fuzzy mathematical morphologies. Fuzzy Sets and Systems, Vol. 124, 73-85, 2001.

[51] H.T. Nguyen, Y. Wang, G. Wei. On Choquet theorem for random upper semicontinuous functions. International Journal of Approximate Reasoning, Vol. 46, Issue 1, 3-16, 2007. 
[52] S. Osher, J. Sethian. Fronts Propagating with Curvature-Dependent Speed: Algorithms Based on Hamilton-Jacobi Formulations. Journal of Computational Physics, 79, 12-49, 1988.

[53] J.-P. Penot, C. Zălinescu. Approximation of Functions and Sets. In (M. Lassonde, Ed.) Approximation, Optimization and Mathematical Economics, 255-274, Physica-Verlag Heidelberg, 2001.

[54] F. Préteux, M. Schmitt. Boolean Texture Analysis and Synthesis. In (J. Serra, Ed.) Image Analysis and Mathematical Morphology, Vol. 2, Chapter 18, Academic Press, 1988.

[55] R.T. Rockafellar. Convex analysis. Princeton University Press, Princeton NJ, 1970.

[56] E. Rouy, A. Tourin. A Viscosity Solutions Approach to Shape from Shading. SIAM Journal on Numerical Analysis, Vol. 29, No. 3, 867-884, 1992.

[57] A. Seeger, M. Volle. On a convolution operation obtained by adding level sets: classical and new results. Operations Research, Vol. 29, No. 2, 131-154, 1995.

[58] J. Serra. Image Analysis and Mathematical Morphology. Academic Press, London, 1982.

[59] J. Serra. Image Analysis and Mathematical Morphology. Vol II: Theoretical Advances, Academic Press, London, 1988.

[60] J. Serra. Boolean Random Functions. Journal of Microscopy, Vol 156, pt. 1, 41-63.

[61] M. Schmitt. Support Function and Minkowski Addition of Non-Convex Sets. In (P. Maragos, R. Shafer, M. Butt, Eds.) Mathematical Morphology and its Applications to image and signal processing, Kluwer, 15-22, 1996.

[62] P. Soille. Morphological Image Analysis, Springer-Verlag, Berlin, 1999.

[63] C. Vachier, F. Meyer. The Viscous Watershed Transform. Journal of Mathematical Imaging and Vision, Vol. 22, 251-267, 2005.

[64] C. Vachier, F. Meyer. News from Viscous Land. In Proc. of 8th Internation Symposium on Mathematical Morphology (ISMM'07), pp. 189-200., Rio de Janeiro, Brazil, 2007.

[65] T.D. Van and N.D.T. Son. Hopf-Lax-Oleinik-Type Estimates for Viscosity Solutions to Hamilton-Jacobi Equations with Concave-Convex Data. Vietnam Journal of Mathematics, Vol. 34, No. 2, 209-239, 2006.

[66] L. Vincent. Morphological grayscale reconstruction in image analysis: applications and efficient algorithms. IEEE Trans. on Image Processing, Vol. 2, Issue 2, 176-201, 1993. 
[67] M. Volle. The Use of Monotone Norms in Epigraphical Analysis. Journal of Convex Analysis, Vol. 1, No. 2, 203-224, 1994

[68] M. Volle. Duality for the Level Sum of Quasiconvex Functions and Applications. ESAIM: Control, Optimisation and Calculus of Variations, Vol. 3, 329-343, 1998.

[69] G. Wei, Y. Wang. On metrization of the hit-or-miss topology using Alexandroff compactification. International Journal of Approximate Reasoning, Vol. 46, Issue 1, 47-64, 2007. 